\title{
KÄHLER MANIFOLDS OF QUASI-CONSTANT HOLOMORPHIC SECTIONAL CURVATURES
}

\author{
G. GANCHEV AND V. MIHOVA
}

\begin{abstract}
The Kähler manifolds of quasi-constant holomorphic sectional curvatures are introduced as Kähler manifolds with complex distribution of codimension two, whose holomorphic sectional curvature only depends on the corresponding point and the geometric angle, associated with the section. A curvature identity characterizing such manifolds is found. The biconformal group of transformations whose elements transform Kähler metrics into Kähler ones is introduced and biconformal tensor invariants are obtained. This makes it possible to classify the manifolds under consideration locally. The class of locally biconformal flat Kähler metrics is shown to be exactly the class of Kähler metrics whose potential function is only a function of the distance from the origin in $\mathbb{C}^{n}$. Finally we show that any rotational even dimensional hypersurface carries locally a natural Kähler structure which is of quasi-constant holomorphic sectional curvatures.
\end{abstract}

\section{INTRODUCTION}

In the present paper we study Kähler manifolds $(M, g, J, D)(\operatorname{dim} M=2 n)$ with $J$ invariant distribution $D$ of $\operatorname{dim} D=2(n-1)$. The orthogonal distribution $D^{\perp}$ of the given distribution $D$ is also $J$-invariant and $\operatorname{dim} D^{\perp}=2$. The structural group of these manifolds is $U(n-1) \times U(1)$.

For example any real function $u \in C^{\infty}$ on $M$ with $d u \neq 0$ generates a $J$-invariant distribution $D$ with $D^{\perp}=\operatorname{span}\{\operatorname{grad} u, J g r a d u\}$. Thus any Kähler manifold with scalar curvature $\tau$ in general carries such a structure $\left(D, D^{\perp}\right)$ generated by $\operatorname{grad} \tau$ and $\operatorname{Jgrad} \tau$. In this case the structure $\left(D, D^{\perp}\right)$ is determined by the Kähler structure $(g, J)$.

In Section 2 we introduce the basic notion of a Kähler manifold $(M, g, J, D)(n \geq 2)$ with quasi-constant holomorphic sectional curvatures (Kähler $Q C H$-manifolds).

Any holomorphic tangent section at a point $p \in M$ and $D^{\perp}(p)$ determines a geometric angle $\varphi$. Then the notion of a Kähler manifold of quasi-constant holomorphic sectional curvatures is introduced by the natural condition:

The holomorphic sectional curvatures of the manifold $(M, g, J, D)$ depend only on the angle $\varphi$ and the point $p \in M$.

The notion of a Kähler $Q C H$-manifold is the Kähler analogue of the notion of a Riemannian manifold of quasi-constant sectional curvatures [1, 5].

1991 Mathematics Subject Classification. Primary 53B35, Secondary 53C55.

Key words and phrases. Kähler manifolds with $J$-invariant distributions, Kähler manifolds of quasiconstant holomorphic sectional curvatures, biconformal transformations, biconformal invariants, even dimensional rotational hypersurfaces. 
We construct three Kähler tensors $\pi, \Phi$ and $\Psi$ invariant under the action of the structural group and prove Proposition 2.3, which gives the main tool to investigate the Kähler $Q C H$-manifolds:

A Kähler manifold $(M, g, J, D)$ is of quasi-constant holomorphic sectional curvatures if and only if its curvature tensor $R$ satisfies the identity

$$
R=a \pi+b \Phi+c \Psi
$$

where $a, b$ and $c$ are functions on $M$.

In Section 3 we study the integrability conditions for the curvature identity (1.1). A complete system of integrability conditions for (1.1) is obtained in Theorem 3.5. Applying this theorem we obtain:

If the distribution D of a Kähler QCH-manifold is not involutive, then the integrability conditions of (1.1) reduce to the conditions (3.21).

In Section 4 we discuss transformations of Kähler metrics into Kähler ones. By using the induced metric $\eta \otimes \eta+\tilde{\eta} \otimes \tilde{\eta}$ on $D^{\perp}$, we introduce biconformal transformations of the structure $(g, \eta)$ by the formulas

$$
g^{\prime}=e^{2 u}\left\{g+\left(e^{2 v}-1\right)(\eta \otimes \eta+\tilde{\eta} \otimes \tilde{\eta})\right\}, \quad \eta^{\prime}=e^{u+v} \eta,
$$

where $u$ and $v$ are functions on $M$ satisfying certain conditions.

We introduce the notion of a $B$-distribution with the conditions (4.15) and prove that:

All metrics $g^{\prime}$ given by (1.2) are Kählerian if and only if the distribution $D$ is a $B$ distribution.

Further we define $B_{0}$-distributions as a special case of $B$-distributions with the conditions (4.16). In Proposition 4.7 we show that the transformations (1.2) form a group the group of biconformal transformations.

Section 5 is devoted to the tensor invariants of the biconformal group in the case of a $B_{0}$-distribution $D$. Theorem 5.7 states as follows:

The tensor $R-a \pi-b \Phi-c \Psi$ of type $(1,3)$ is a biconformal invariant.

This theorem makes possible a local classification of the Kähler $Q C H$-manifolds satisfying a certain inequality (Theorem 5.11): if

A Kähler manifold $(M, g, J, D)$ with $B_{0}$-distribution $D$ is biconformally flat if and only

$$
R=a \pi+b \Phi+c \Psi, \quad a+k^{2}>0 .
$$

The main property, which connects the Kähler QCH-manifolds with the set of all Kähler metrics, whose potential function is of the type $f\left(r^{2}\right)(r$ - the distance from the origin in $\left.\mathbb{C}^{n}\right)$ is established by Theorem 5.13 .

Any Kähler metric $g=\partial \bar{\partial} f\left(r^{2}\right)$ is biconformally flat and vice versa.

In Section 6 we show that any rotational hypersurface $\left(M^{2 n}, \bar{g}\right)$ in $\mathbb{C}^{n} \times \mathbb{R}$ with axis of revolution $l=\mathbb{R}$, which has no common points with $l$ carries a geometrically determined complex structure $J$. Thus $\left(M^{2 n}, \bar{g}, J\right)$ can be considered as a locally conformal Kähler manifold in all dimensions $2 n \geq 4$. 
Further we introduce a natural Kähler metric on $\left(M^{2 n}, \bar{g}, J\right)$ by the formula (6.16). This formula makes the class of rotational hypersurfaces an important source of Kähler metrics because of Theorem 6.2.

Let $\left(M^{2 n}, \bar{g}, J, \bar{\xi}\right)(2 n \geq 4)$ be a rotational hypersurface satisfying the conditions (6.15). Then the complex dilatational Kähler metric g given by (6.16) is of quasi-constant holomorphic sectional curvatures.

Finally we find the rotational hypersurfaces $M^{2 n}$ whose complex dilatational Kähler metric is of constant holomorphic sectional curvatures:

Any rotational hypersurface $M^{2 n}$ which carries a complex dilatational Kähler metric of constant holomorphic sectional curvature $a=$ const $>0$ is generated by a meridian of the type

$$
\gamma: y= \pm \frac{1}{\sqrt{a}}\left(\sqrt{8-a x^{2}}+\ln \frac{\sqrt{8-a x^{2}}-2}{\sqrt{8-a x^{2}}+2}\right)+y_{0}, \quad 0<x<\frac{2}{\sqrt{a}},
$$

where the meridian $\gamma$ is considered with respect to the usual coordinate system Oxy with axis of revolution $l=O y$.

\section{A tensor CHARACTERIZATION OF THE KÄHLER MANIFOLDS OF QUASI-CONSTANT HOLOMORPHIC SECTIONAL CURVATURES}

Let $(M, g, J, D)$ be a 2 n-dimensional Kähler manifold with metric $g$, complex structure $J$ and $J$-invariant distribution $D$ of codimension 2. The Lie algebra of all $C^{\infty}$ vector fields on $M$ will be denoted by $\mathfrak{X} M$ and $T_{p} M$ will stand for the tangent space to $M$ at any point $p \in M$. In the presence of the distribution $D$ the structure of any tangent space is $T_{p} M=D(p) \oplus D^{\perp}(p)$, where $D^{\perp}(p)$ is the 2-dimensional $J$-invariant orthogonal complement to the space $D(p)$. This means that the structural group of the manifolds under consideration is the subgroup $U(n-1) \times U(1)$ of $U(n)$.

As our considerations are local, we can assume the existence of a unit vector field $\xi$ on $M$ such that $D^{\perp}(p)=\operatorname{span}\{\xi, J \xi\}$ at any point $p \in M$. We denote by $\eta$ and $\tilde{\eta}$ the unit 1-forms corresponding to $\xi$ and $J \xi$, respectively, i.e.

$$
\eta(X)=g(\xi, X), \quad \tilde{\eta}(X)=g(J \xi, X)=-\eta(J X) ; \quad X \in \mathfrak{X} M .
$$

Then the distribution $D$ is determined by the conditions

$$
D(p)=\left\{X \in T_{p} M \mid \eta(X)=\tilde{\eta}(X)=0\right\}, \quad p \in M .
$$

As a rule, we use the following denotations for vector fields (vectors):

$$
X, Y, Z \in \mathfrak{X} M\left(T_{p} M\right) ; \quad x_{0}, y_{0}, z_{0} \in \mathfrak{X} D(D(p)) .
$$

The Kähler form $\Omega$ of the structure $(g, J)$ is given by $\Omega(X, Y)=g(J X, Y), X, Y \in \mathfrak{X} M$.

Let $\nabla$ be the Levi-Civita connection of the metric $g$. The Riemannian curvature tensor $R$, the Ricci tensor $\rho$ and the scalar curvature $\tau$ of $\nabla$ are given by the equalities

$$
\begin{gathered}
R(X, Y) Z=\nabla_{X} \nabla_{Y} Z-\nabla_{Y} \nabla_{X} Z-\nabla_{[X, Y]} Z, \\
R(X, Y, Z, U)=g(R(X, Y) Z, U) ; \quad X, Y, Z, U \in \mathfrak{X} M,
\end{gathered}
$$




$$
\begin{gathered}
\rho(Y, Z)=\sum_{i=1}^{2 n} R\left(e_{i}, Y, Z, e_{i}\right) ; \quad Y, Z \in T_{p} M, \\
\tau=\sum_{i=1}^{2 n} \rho\left(e_{i}, e_{i}\right),
\end{gathered}
$$

where $\left\{e_{i}\right\}, i=1, \ldots, 2 n$ is an orthonormal basis for $T_{p} M, p \in M$.

We recall that the curvature tensor $R$ of any Kähler manifold satisfies the identities

$$
\begin{aligned}
& R(X, Y, Z, U)=-R(Y, X, Z, U) \\
& R(X, Y) Z+R(Y, Z) X+R(Z, X) Y=0 \\
& R(X, Y, Z, U)=-R(X, Y, U, Z) \\
& R(X, Y) J Z=J R(X, Y) Z
\end{aligned}
$$

Now we shall introduce the geometric functions and tensors associated with the structures $(g, J, D)$.

All directions in $\operatorname{span}\{\xi, J \xi\}$ have one and the same Ricci curvature which is denoted by $\sigma$, i. e.

$$
\sigma=\rho(\xi, \xi)=\rho(J \xi, J \xi)
$$

The Riemannian sectional curvature of the structural distribution $D^{\perp}$ is denoted by $\varkappa$, i. e.

$$
\varkappa=R(\xi, J \xi, J \xi, \xi) .
$$

Thus the structures $(g, J, D)$ give rise to the functions $\varkappa, \sigma$ and $\tau$.

Further we note that the tensor $\eta(X) \eta(Y)+\tilde{\eta}(X) \tilde{\eta}(Y)$ does not depend on the basis $\{\xi, J \xi\}$. Then the fundamental symmetric tensors of type $(0,2)$ are

$$
g(X, Y), \quad \eta(X) \eta(Y)+\tilde{\eta}(X) \tilde{\eta}(Y) ; \quad X, Y \in \mathfrak{X} M
$$

We also mention the corresponding fundamental skew symmetric tensors

$$
\Omega(X, Y), \quad \eta(X) \tilde{\eta}(Y)-\eta(Y) \tilde{\eta}(X) ; \quad X, Y \in \mathfrak{X} M .
$$

Any tensor over $T_{p} M, p \in M$ of type $(0,4)$ having the symmetries $(2.1)$ is called a Kähler tensor. We need the following invariant Kähler tensors:

$$
\begin{aligned}
4 \pi(X, Y, Z, U)= & g(Y, Z) g(X, U)-g(X, Z) g(Y, U) \\
& +g(J Y, Z) g(J X, U)-g(J X, Z) g(J Y, U) \\
& -2 g(J X, Y) g(J Z, U) ;
\end{aligned}
$$




$$
\begin{aligned}
& 8 \Phi(X, Y, Z, U)= g(Y, Z)\{\eta(X) \eta(U)+\tilde{\eta}(X) \tilde{\eta}(U)\} \\
&-g(X, Z)\{\eta(Y) \eta(U)+\tilde{\eta}(Y) \tilde{\eta}(U)\} \\
&+g(X, U)\{\eta(Y) \eta(Z)+\tilde{\eta}(Y) \tilde{\eta}(Z)\} \\
&-g(Y, U)\{\eta(X) \eta(Z)+\tilde{\eta}(X) \tilde{\eta}(Z)\} \\
&+g(J Y, Z)\{\eta(X) \tilde{\eta}(U)-\eta(U) \tilde{\eta}(X)\} \\
&-g(J X, Z)\{\eta(Y) \tilde{\eta}(U)-\eta(U) \tilde{\eta}(Y)\} \\
&+g(J X, U)\{\eta(Y) \tilde{\eta}(Z)-\eta(Z) \tilde{\eta}(Y)\} \\
&-g(J Y, U)\{\eta(X) \tilde{\eta}(Z)-\eta(Z) \tilde{\eta}(X)\} \\
&-2 g(J X, Y)\{\eta(Z) \tilde{\eta}(U)-\eta(U) \tilde{\eta}(Z)\} \\
&-2 g(J Z, U)\{\eta(X) \tilde{\eta}(Y)-\eta(Y) \tilde{\eta}(X)\} ; \\
& \eta(Y) \eta(Z) \tilde{\eta}(X) \tilde{\eta}(U)-\eta(X) \eta(Z) \tilde{\eta}(Y) \tilde{\eta}(U) \\
&+ \eta(X) \eta(U) \tilde{\eta}(Y) \tilde{\eta}(Z)-\eta(Y) \eta(U) \tilde{\eta}(X) \tilde{\eta}(Z) \\
& \Psi(X, Y, Z, U)=\{(\eta \tilde{\eta}) \otimes(\tilde{\eta} \wedge \eta)\}(X, Y, Z, U),
\end{aligned}
$$

$X, Y, Z, U \in \mathfrak{X} M$.

These tensors are invariant under the action of the structural group $U(n-1) \times U(1)$ on the tensors over $T_{p} M, p \in M$ in the standard sense (e.g. [11]).

Let $(M, g, J, D)(\operatorname{dim} M=2 n)$ be a Kähler manifold with J-invariant distributions $D(\operatorname{dim} D=2(n-1))$ and $D^{\perp}$. The structures $(g, J, D)$ give rise to the following geometric types of sectional curvatures with respect to any Kähler curvature tensor (e. g. $R$ ):

horizontal sectional curvatures $R\left(x_{0}, y_{0}, y_{0}, x_{0}\right)$, where $\left\{x_{0}, y_{0}\right\}$ is any orthonormal pair in $D$;

mixed sectional curvatures $R\left(x_{0}, e, e, x_{0}\right)$, where $x_{0}$ and $e$ are unit vectors in $D$ and $D^{\perp}$, respectively;

vertical sectional curvature $R(e, J e, J e, e)$, where $e$ is a unit vector in $D^{\perp}$.

Let $\left\{e_{i}\right\}, i=1, \ldots, 2(n-1)$ and $\{e, J e\}$ be orthonormal bases at a point $p \in M$ of $D$ and $D^{\perp}$, respectively. Then there arise three types of scalar curvatures associated with the curvature tensor $R$ :

horizontal scalar curvature

$$
\sum_{i, j=1}^{2(n-1)} R\left(e_{i}, e_{j}, e_{j}, e_{i}\right)=\tau-2 \sigma-2(\sigma-\varkappa)
$$

mixed scalar curvature

$$
\sum_{i=1}^{2(n-1)} R\left(e_{i}, e, e, e_{i}\right)=\sigma-\varkappa
$$

vertical scalar curvature

$$
R(e, J e, J e, e)=\varkappa .
$$


The invariant Kähler tensors

$$
\pi-2 \Phi+\Psi, \quad \Phi-\Psi, \quad \Psi
$$

are closely related to the above geometric types of sectional curvatures because of the following their properties:

all geometric sectional curvatures of $\pi-2 \Phi+\Psi$ are zero except its horizontal sectional curvatures (more precisely this tensor is of constant horizontal holomorphic sectional curvatures);

all geometric sectional curvatures of $\Phi-\Psi$ are zero except its mixed sectional curvatures (more precisely this tensor is of constant mixed sectional curvatures);

all geometric sectional curvatures of $\Psi$ are zero except its vertical sectional curvature.

In this section we deal with holomorphic sectional curvatures taking into account the structure of the tangent space at any point of $M$.

Any vector field $X \in \mathfrak{X} M$ is decomposable in a unique way as follows:

$$
X=x_{0}+\tilde{\eta}(X) J \xi+\eta(X) \xi,
$$

where $x_{0}$ is the projection of $X$ into $\mathfrak{X} D$.

Let $\operatorname{span}\{X, J X\}$ be an arbitrary holomorphic 2-plane (section) in the tangent space $T_{p} M, p \in M$ generated by the unit vector $X$. For any unit vector $Y \in \operatorname{span}\{X, J X\}$ we have

$$
\eta^{2}(Y)+\tilde{\eta}^{2}(Y)=\eta^{2}(X)+\tilde{\eta}^{2}(X) .
$$

The holomorphic section $\operatorname{span}\{X, J X\}$ and the structural section $\operatorname{span}\{\xi, J \xi\}=D^{\perp}(p)$ form an angle

$$
\varphi=\angle(\operatorname{span}\{X, J X\}, \operatorname{span}\{\xi, J \xi\}), \quad \varphi \in\left[0, \frac{\pi}{2}\right]
$$

which is uniquely determined by the equality

$$
\cos ^{2} \varphi=\eta^{2}(X)+\tilde{\eta}^{2}(X)
$$

This geometric angle measures the deviation of any holomorphic section from the structural tangent plane $D^{\perp}$.

Now we can give the basic definition in our considerations.

Definition 2.1. Let $(M, g, J, D)$ be a Kähler manifold with $\operatorname{dim} M=2 n \geq 4$ and $J$ invariant distribution $D$ of codimension 2. The manifold is said to be of quasi-constant holomorphic sectional curvatures (a Kähler QCH-manifold) if for any holomorphic section $\operatorname{span}\{X, J X\}$ generated by the unit tangent vector $X \in T_{p} M, p \in M$ with $\varphi=$ $\angle(\operatorname{span}\{X, J X\}, \operatorname{span}\{\xi, J \xi\})$ the Riemannian sectional curvature $R(X, J X, J X, X)$ may only depend on the point $p \in M$ and the angle $\varphi$, i. e.

$$
R(X, J X, J X, X)=f(p, \varphi), \quad p \in M, \varphi \in\left[0, \frac{\pi}{2}\right] .
$$

This notion corresponds to the notion of a Riemannian manifold of quasi-constant sectional curvature (cf [1, 5]).

The first essential step in the study of Kähler $Q C H$-manifolds is to find a curvature identity characterizing these manifolds. 
Lemma 2.2. Let $(M, g, J, D)(\operatorname{dim} M=2 n \geq 4)$ be a Kähler manifold of quasi-constant holomorphic sectional curvatures. Then the curvature tensor $R$ satisfies the following equalities

$$
\begin{aligned}
& \text { i) } R\left(x_{0}, J x_{0}, J x_{0}, \xi\right)=R\left(\xi, J \xi, J \xi, x_{0}\right)=0 \\
& \text { ii) } R\left(x_{0}, J x_{0}, x_{0}, \xi\right)=R\left(\xi, J \xi, x_{0}, \xi\right)=0 ; \\
& \text { iii) } R\left(x_{0}, J \xi, J \xi, x_{0}\right)=R\left(x_{0}, \xi, \xi, x_{0}\right)=\frac{1}{2} R\left(x_{0}, J x_{0}, J \xi, \xi\right)
\end{aligned}
$$

for all unit vectors $x_{0} \in D$.

Proof. Let $x_{0}$ be an arbitrary unit vector in $D$.

i) For any $t>0$ we set $X^{\prime}=\frac{x_{0}+t \xi}{\sqrt{1+t^{2}}}, X^{\prime \prime}=\frac{-x_{0}+t \xi}{\sqrt{1+t^{2}}}$. The unit vectors $X^{\prime}$ and $X^{\prime \prime}$ give rise to the sections $\operatorname{span}\left\{X^{\prime}, J X^{\prime}\right\}$ and $\operatorname{span}\left\{X^{\prime \prime}, J X^{\prime \prime}\right\}$ which form one and the same angle $\varphi$ with $\operatorname{span}\{\xi, J \xi\}$ given by the equality

$$
\cos \varphi=\frac{t}{\sqrt{1+t^{2}}}
$$

Hence $R\left(X^{\prime}, J X^{\prime}, J X^{\prime}, X^{\prime}\right)=R\left(X^{\prime \prime}, J X^{\prime \prime}, J X^{\prime \prime}, X^{\prime \prime}\right)$. Replacing $X^{\prime}$ and $X^{\prime \prime}$ in the last equality we obtain

$$
A^{\prime}+A^{\prime \prime}=A^{\prime}-A^{\prime \prime}
$$

where

$$
\begin{gathered}
A^{\prime}=R\left(x_{0}, J x_{0}, J x_{0}, x_{0}\right)+2 t^{2}\left\{R\left(x_{0}, \xi, \xi, x_{0}\right)+3 R\left(x_{0}, J \xi, J \xi, x_{0}\right)\right\}+t^{4} R(\xi, J \xi, J \xi, \xi), \\
A^{\prime \prime}=4 t\left\{R\left(x_{0}, J x_{0}, J x_{0}, \xi\right)+t^{2} R\left(\xi, J \xi, J \xi, x_{0}\right)\right\} .
\end{gathered}
$$

The last equality and (2.12) imply i).

ii) In this case we consider the unit vectors $Y^{\prime}=\frac{x_{0}+t J \xi}{\sqrt{1+t^{2}}}, Y^{\prime \prime}=\frac{-x_{0}+t J \xi}{\sqrt{1+t^{2}}}, t>$ 0 . The holomorphic sections $\operatorname{span}\left\{Y^{\prime}, J Y^{\prime}\right\}$ and $\operatorname{span}\left\{Y^{\prime \prime}, J Y^{\prime \prime}\right\}$ form the same angle $\varphi$ given by (2.11) with the section $\operatorname{span}\{\xi, J \xi\}$. Replacing $Y^{\prime}$ and $Y^{\prime \prime}$ in the equality $R\left(Y^{\prime}, J Y^{\prime}, J Y^{\prime}, Y^{\prime}\right)=R\left(Y^{\prime \prime}, J Y^{\prime \prime}, J Y^{\prime \prime}, Y^{\prime \prime}\right)$ we find

$$
B^{\prime}-B^{\prime \prime}=B^{\prime}+B^{\prime \prime},
$$

where

$$
\begin{gathered}
B^{\prime}=R\left(x_{0}, J x_{0}, J x_{0}, x_{0}\right)+2 t^{2}\left\{R\left(x_{0}, J \xi, J \xi, x_{0}\right)+3 R\left(x_{0}, \xi, \xi, x_{0}\right)\right\}+t^{4} R(\xi, J \xi, J \xi, \xi), \\
B^{\prime \prime}=4 t\left\{R\left(x_{0}, J x_{0}, x_{0}, \xi\right)+t^{2} R\left(\xi, J \xi, x_{0}, \xi\right)\right\} .
\end{gathered}
$$

The last equality and (2.13) imply ii).

iii) As the holomorphic sections $\operatorname{span}\left\{X^{\prime}, J X^{\prime}\right\}$ and $\operatorname{span}\left\{Y^{\prime}, J Y^{\prime}\right\}$ form the same angle $\varphi$ with the section $\operatorname{span}\{\xi, J \xi\}$, their sectional curvatures are equal. This condition implies $A^{\prime}=B^{\prime}$ and $R\left(x_{0}, J \xi, J \xi, x_{0}\right)=R\left(x_{0}, \xi, \xi, x_{0}\right)$. Further, the last equality in iii) follows from the identity

$$
R\left(x_{0}, J x_{0}, J \xi, \xi\right)=R\left(x_{0}, \xi, \xi, x_{0}\right)+R\left(x_{0}, J \xi, J \xi, x_{0}\right) .
$$


The curvature identity characterizing Kähler manifolds of quasi-constant holomorphic sectional curvatures is given by the following

Proposition 2.3. Let $(M, g, J, D)(\operatorname{dim} M=2 n \geq 4)$ be a Kähler manifold with Jinvariant $2(n-1)$-dimensional distribution $D$. Then $(M, g, J, D)$ is of quasi-constant holomorphic sectional curvatures if and only if

$$
R=a \pi+b \Phi+c \Psi
$$

where $a, b$ and $c$ are functions on $M$ and the tensors $\pi, \Phi$ and $\Psi$ are given by $(2.4),(2.5)$ and (2.6), respectively.

Proof. Let $(M, g, J, D)$ be a Kähler manifold of quasi-constant holomorphic sectional curvatures $f(p, \varphi)$ and $T_{p} M, p \in M$ be the tangent space to $M$ at a fixed point $p \in M$. Consider a unit vector $X \in T_{p} M$ which is neither in $D$ nor in $D^{\perp}$, i.e. the angle $\varphi$ between $\operatorname{span}\{X, J X\}$ and $\operatorname{span}\{\xi, J \xi\}$ is in the interval $\left(0, \frac{\pi}{2}\right)$. It is an easy verification that there exists a unique unit vector $x$ in $\operatorname{span}\{X, J X\}$ so that $x \perp \xi$ and $\tilde{\eta}(x)=\cos \varphi$. Then we have

$$
x=\sin \varphi x_{0}+\cos \varphi J \xi, \quad x_{0} \in D, \quad\left\|x_{0}\right\|=1
$$

and

$$
R(X, J X, J X, X)=R(x, J x, J x, x) .
$$

Now we replace $x$ from (2.14) into the last equality, linearize and taking into account Lemma 2.2 we obtain

$$
\begin{aligned}
& R(X, J X, J X, X)= \\
& \sin ^{4} \varphi R\left(x_{0}, J x_{0}, J x_{0}, x_{0}\right)+8 \sin ^{2} \varphi \cos ^{2} \varphi R\left(x_{0}, \xi, \xi, x_{0}\right)+\cos ^{4} \varphi R(\xi, J \xi, J \xi, \xi) .
\end{aligned}
$$

This equality can be written as

$$
\begin{aligned}
& R(X, J X, J X, X)= \\
& \sin ^{4} \varphi f\left(p, \frac{\pi}{2}\right)+\cos ^{4} \varphi f(p, 0)+8 \sin ^{2} \varphi \cos ^{2} \varphi R\left(x_{0}, \xi, \xi, x_{0}\right) .
\end{aligned}
$$

Further we shall express the sectional curvature $R\left(x_{0}, \xi, \xi, x_{0}\right)$ by the functions $f(p, 0), f\left(p, \frac{\pi}{2}\right)$ and $f\left(p, \frac{\pi}{4}\right)$.

Let us consider the unit vector $X^{\prime}=\frac{x_{0}+\xi}{\sqrt{2}}$. We note that the angle between $\operatorname{span}\left\{X^{\prime}, J X^{\prime}\right\}$ and $\operatorname{span}\{\xi, J \xi\}$ is $\frac{\pi}{4}$. Replacing $X^{\prime}$ into the equality

$$
f\left(p, \frac{\pi}{4}\right)=R\left(X^{\prime}, J X^{\prime}, J X^{\prime}, X^{\prime}\right)
$$

we obtain

$$
8 R\left(x_{0}, \xi, \xi, x_{0}\right)=4 f\left(p, \frac{\pi}{4}\right)-f\left(p, \frac{\pi}{2}\right)-f(p, 0) .
$$

Now the last equality and (2.15) imply

$$
\begin{aligned}
R(X, J X, J X, X)=f( & \left.p, \frac{\pi}{2}\right)+\left\{4 f\left(p, \frac{\pi}{4}\right)-3 f\left(p, \frac{\pi}{2}\right)-f(p, 0)\right\} \cos ^{2} \varphi \\
& +\left\{2 f\left(p, \frac{\pi}{2}\right)+2 f(p, 0)-4 f\left(p, \frac{\pi}{4}\right)\right\} \cos ^{4} \varphi
\end{aligned}
$$


Putting

we obtain

$$
\begin{gathered}
a(p)=f\left(p, \frac{\pi}{2}\right), b(p)=4 f\left(p, \frac{\pi}{4}\right)-3 f\left(p, \frac{\pi}{2}\right)-f(p, 0), \\
c(p)=2 f\left(p, \frac{\pi}{2}\right)+2 f(p, 0)-4 f\left(p, \frac{\pi}{4}\right)
\end{gathered}
$$

$$
R(X, J X, J X, X)=a+b \cos ^{2} \varphi+c \cos ^{4} \varphi .
$$

We note that the last equality is also true for the boundary angles $\varphi=0, \frac{\pi}{2}$.

On the other hand the holomorphic sectional curvatures of the tensor $a \pi+b \Phi+c \Psi$ are the same as those given in (2.16). The standard lemma for Kähler tensors states that if two Kähler tensors have the same holomorphic sectional curvatures, they coincide (e.g. 7]).

Thus we obtained that

$$
R=a \pi+b \Phi+c \Psi
$$

and

$$
f(p, \varphi)=a+b \cos ^{2} \varphi+c \cos ^{4} \varphi .
$$

The inverse is an immediate verification.

Proposition 2.3 implies in a straightforward way

Corollary 2.4. If $(M, g, J, D)(\operatorname{dim} M=2 n \geq 4)$ is a Kähler manifold of quasi-constant holomorphic sectional curvatures, then its Ricci tensor $\rho$ satisfies the following identity

$$
\rho=\frac{\tau-2 \sigma}{2(n-1)} g+\frac{2 n \sigma-\tau}{2(n-1)}(\eta \otimes \eta+\tilde{\eta} \otimes \tilde{\eta}) .
$$

Taking into account Proposition 2.3 and Corollary 2.4 we obtain expressions for the functions $a, b$ and $c$ by the geometric functions $\varkappa, \sigma$ and $\tau$ :

$$
\begin{aligned}
& a=\frac{\tau-4 \sigma+2 \varkappa}{n(n-1)}, \quad b=\frac{4(n+2) \sigma-2 \tau-4(n+1) \varkappa}{n(n-1)}, \\
& c=\frac{\tau-4(n+1) \sigma+(n+1)(n+2) \varkappa}{n(n-1)} .
\end{aligned}
$$

It follows from Proposition 2.3 that the curvature tensor $R$ of any Kähler $Q C H$-manifold can be represented by the tensors (2.10) in the form

$$
R=a(\pi-2 \Phi+\Psi)+(2 a+b)(\Phi-\Psi)+(a+b+c) \Psi .
$$

The last formula shows that the curvature tensor $R$ of any Kähler $Q C H$-manifold has:

pointwise constant horizontal holomorphic sectional curvatures

$$
R\left(x_{0}, J x_{0}, J x_{0}, x_{0}\right)=a=\frac{\tau-2 \sigma}{n(n-1)}-2 \frac{\sigma-\varkappa}{n(n-1)}, \quad x_{0} \in D,\left\|x_{0}\right\|=1 ;
$$

pointwise constant mixed sectional curvatures

$$
R\left(x_{0}, e, e, x_{0}\right)=\frac{2 a+b}{8}=\frac{\sigma-\varkappa}{2(n-1)}, x_{0} \in D, e \in D^{\perp},\left\|x_{0}\right\|=\|e\|=1 ;
$$


vertical sectional curvature

$$
R(e, J e, J e, e)=a+b+c=\varkappa, \quad e \in D^{\perp},\|e\|=1 .
$$

Remark 2.5. Let $(M, g, J, D)(\operatorname{dim} M=2 n \geq 4)$ be a Kähler manifold with $J$-invariant distributions $D(\operatorname{dim} D=2(n-1))$ and $D^{\perp}$. Then the structures $(g, J, D)$ generate the functions $a, b$ and $c$ by the equalities (2.17) and these functions are related to the scalar curvatures (2.7), (2.8) and (2.9) as follows:

$$
\begin{gathered}
\tau-2 \sigma-2(\sigma-\varkappa)=n(n-1) a, \\
\sigma-\varkappa=\frac{n-1}{4}(2 a+b), \\
\varkappa=a+b+c .
\end{gathered}
$$

\section{INTEGRABILITY CONDITIONS FOR KÄHLER MANIFOLDS OF QUASI-CONSTANT HOLOMORPHIC SECTIONAL CURVATURES}

The next essential step in the study of the Kähler $Q C H$-manifolds is to investigate the integrability conditions following from the identity

$$
R=a \pi+b \Phi+c \Psi .
$$

First we shall make some general notes about the structural distributions $D$ and $D^{\perp}$.

If $D^{\perp}=\operatorname{span}\{\xi, J \xi\}$, we introduce the relative divergences $d i v_{0} \xi$ and $d i v_{0} J \xi$ (relative codifferentials $\delta_{0} \eta$ and $\delta_{0} \tilde{\eta}$ ) of the vector fields $\xi$ and $J \xi$ (1-forms $\eta$ and $\tilde{\eta}$ ) with respect to the distribution $D$ :

$$
d i v_{0} \xi=-\delta_{0} \eta=\sum_{i=1}^{2(n-1)}\left(\nabla_{e_{i}} \eta\right) e_{i}, \quad \operatorname{div} v_{0} J \xi=-\delta_{0} \tilde{\eta}=\sum_{i=1}^{2(n-1)}\left(\nabla_{e_{i}} \tilde{\eta}\right) e_{i},
$$

where $\left\{e_{1}, \ldots, e_{2(n-1)}\right\}$ is an orthonormal basis of $D(p), p \in M$.

Lemma 3.1. Let $(M, g, J, D)$ be a Kähler manifold with J-invariant distribution $D$ of codimension two and $D^{\perp}=\operatorname{span}\{\xi, J \xi\}$. Then

i) the function $\left(\delta_{0} \eta\right)^{2}+\left(\delta_{0} \tilde{\eta}\right)^{2}$ does not depend on the frame field $\{\xi, J \xi\}$;

ii) at the points $p \in M$, where $\left(\delta_{0} \eta\right)^{2}+\left(\delta_{0} \tilde{\eta}\right)^{2}>0$, there exists a geometrically determined frame field

of $D^{\perp}$.

$$
\left\{\frac{\delta_{0} \eta \xi+\delta_{0} \tilde{\eta} J \xi}{\sqrt{\left(\delta_{0} \eta\right)^{2}+\left(\delta_{0} \tilde{\eta}\right)^{2}}}, \frac{-\delta_{0} \tilde{\eta} \xi+\delta_{0} \eta J \xi}{\sqrt{\left(\delta_{0} \eta\right)^{2}+\left(\delta_{0} \tilde{\eta}\right)^{2}}}\right\}
$$

Proof. Let $\xi^{\prime}=\cos \varphi \xi+\sin \varphi J \xi, \quad J \xi^{\prime}=-\sin \varphi \xi+\cos \varphi J \xi$ be an arbitrary frame field of $D^{\perp}$ and $\eta^{\prime}, \tilde{\eta}^{\prime}$ be the 1 -forms corresponding to $\xi^{\prime}, J \xi^{\prime}$, respectively. Then we find

$$
\begin{aligned}
& \left(\nabla_{x_{0}} \eta^{\prime}\right) y_{0}=\cos \varphi\left(\nabla_{x_{0}} \eta\right) y_{0}+\sin \varphi\left(\nabla_{x_{0}} \tilde{\eta}\right) y_{0}, \\
& \left(\nabla_{x_{0}} \tilde{\eta}^{\prime}\right) y_{0}=-\sin \varphi\left(\nabla_{x_{0}} \eta\right) y_{0}+\cos \varphi\left(\nabla_{x_{0}} \tilde{\eta}\right) y_{0}
\end{aligned}
$$

for all $x_{0}, y_{0} \in D$. 
The above equations immediately imply the statement of the lemma.

QED

We call the geometric frame field in the case ii) of the above lemma the principal frame field for $D^{\perp}$.

In the next calculations we shall use the complexifications $T_{p}^{C} M$ and $D^{C}(p), p \in M$ and their standard splitting

$$
T_{p}^{C} M=T_{p}^{1,0} M \oplus T_{p}^{0,1} M, \quad D^{C}(p)=D^{1,0}(p) \oplus D^{0,1}(p) .
$$

Any complex basis of $T_{p}^{1,0} M$ will be denoted by $\left\{Z_{\alpha}\right\}, \alpha=1, \ldots, n$ and the conjugate basis $\left\{Z_{\bar{\alpha}}=\overline{Z_{\alpha}}\right\}, \bar{\alpha}=\overline{1}, \ldots, \bar{n}$ will span $T_{p}^{0,1} M$. Counting the special structure of the tangent spaces, we shall also consider special complex bases of the type $\left\{Z_{0}, Z_{\lambda}\right\}$, where $Z_{0}=\frac{\xi-i J \xi}{2}$ and $\left\{Z_{\lambda}\right\}, \lambda=1, \ldots, n-1$ form a basis for $D^{1,0}(p)$. Then $\left\{Z_{\overline{0}}, Z_{\bar{\lambda}}\right\}, \bar{\lambda}=$ $\overline{1}, \ldots, \overline{n-1}$ is a special complex basis for $T_{p}^{0,1} M$.

Unless otherwise stated, the Greek indices $\alpha, \beta, \gamma, \delta, \varepsilon$ will run through $1, \ldots, n$, while the Greek indices $\lambda, \mu, \nu, \varkappa, \sigma$ will run through $1, \ldots, n-1$.

First we give the essential components (which may not be zero) of the fundamental tensors $g, \Omega, \eta, \tilde{\eta}$ :

$$
\begin{gathered}
g_{\alpha \bar{\beta}}=g\left(Z_{\alpha}, Z_{\bar{\beta}}\right), \quad \Omega_{\alpha \bar{\beta}}=\Omega\left(Z_{\alpha}, Z_{\bar{\beta}}\right)=i g_{\alpha \bar{\beta}} \\
\eta_{\alpha}=g_{\alpha \overline{0}}, \quad \eta_{\bar{\alpha}}=g_{\bar{\alpha} 0}, \quad \tilde{\eta}_{\alpha}=-i \eta_{\alpha}, \quad \tilde{\eta}_{\bar{\alpha}}=i \eta_{\bar{\alpha}} ; \\
\eta_{\lambda}=\eta_{\bar{\lambda}}=0, \quad \eta_{0}=\eta_{\overline{0}}=g_{0 \overline{0}}=\frac{1}{2}
\end{gathered}
$$

We introduce the following functions and 1-forms associated with the vector fields $\nabla_{\xi} \xi$ and $\nabla_{J \xi} J \xi$ :

$$
\begin{gathered}
p=g\left(\nabla_{\xi} \xi, J \xi\right), \quad p^{*}=g\left(\nabla_{J \xi} J \xi, \xi\right) . \\
\theta(X)=g\left(\nabla_{\xi} \xi, X\right)-p \tilde{\eta}(X), \quad \theta^{*}(X)=g\left(\nabla_{J \xi} J \xi, X\right)-p^{*} \eta(X), \quad X \in T_{p} M .
\end{gathered}
$$

It is clear that $\theta(X)=\theta\left(x_{0}\right), \theta^{*}(X)=\theta^{*}\left(x_{0}\right)$, where $x_{0}=X-\tilde{\eta}(X) J \xi-\eta(X) \xi$.

Taking into account that $Z_{0}=\frac{\xi-i J \xi}{2}, Z_{\overline{0}}=\frac{\xi+i J \xi}{2}$ we find

$$
\begin{gathered}
\nabla_{0} \eta_{\lambda}=\frac{1}{2}\left(\theta_{\lambda}+\theta_{\lambda}^{*}\right), \quad \nabla_{\overline{0}} \eta_{\lambda}=\frac{1}{2}\left(\theta_{\lambda}-\theta_{\lambda}^{*}\right) ; \\
\nabla_{0} \eta_{0}=\frac{p^{*}-i p}{4}, \quad \nabla_{0} \eta_{\overline{0}}=\frac{-p^{*}+i p}{4} .
\end{gathered}
$$

The next two natural statements characterize involutive distributions $D$ and $D^{\perp}$.

Lemma 3.2. The distribution $D$ is involutive if and only if

$$
\nabla_{\lambda} \eta_{\mu}-\nabla_{\mu} \eta_{\lambda}=0, \quad \nabla_{\lambda} \eta_{\bar{\mu}}=0
$$


Proof. Since the distribution $D$ is determined by the 1-forms $\eta$ and $\tilde{\eta}$, it is involutive if and only if

$$
d \eta\left(x_{0}, y_{0}\right)=0, \quad d \tilde{\eta}\left(x_{0}, y_{0}\right)=0, \quad x_{0}, y_{0} \in D .
$$

On the other hand, we have

$$
\begin{gathered}
\nabla_{\lambda} \tilde{\eta}_{\mu}=-i \nabla_{\lambda} \eta_{\mu}, \quad \nabla_{\lambda} \tilde{\eta}_{\bar{\mu}}=i \nabla_{\lambda} \eta_{\bar{\mu}} \\
d \tilde{\eta}_{\lambda \mu}=-i d \eta_{\lambda \mu}, \quad d \tilde{\eta}_{\lambda \bar{\mu}}=i\left(\nabla_{\lambda} \eta_{\bar{\mu}}+\nabla_{\bar{\mu}} \eta_{\lambda}\right) .
\end{gathered}
$$

which imply the lemma.

QED

Lemma 3.3. The distribution $D^{\perp}$ is involutive if and only if

$$
\theta_{\lambda}+\theta_{\lambda}^{*}=0 \text {. }
$$

Proof. The distribution $D^{\perp}$ is involutive if and only if the component of the Lie bracket $[\xi, J \xi]$ in $D$ is zero, i.e.

$$
g\left(\nabla_{\xi} J \xi-\nabla_{J \xi} \xi, x_{0}\right)=0, \quad x_{0} \in D
$$

The last equality is equivalent to the condition $\theta\left(x_{0}\right)+\theta^{*}\left(x_{0}\right)=0, x_{0} \in D$.

QED

Remark 3.4. It is easy to check that the conditions in Lemma 3.2 and Lemma 3.3 do not depend on the frame field $\{\xi, J \xi\}$.

Further we give the essential components of the tensors $\pi, \Phi$ and $\Psi$ with respect to a complex basis $\left\{Z_{\alpha}\right\}, \alpha=1, \ldots, n$ :

$$
\begin{aligned}
\pi_{\alpha \bar{\beta} \gamma \bar{\delta}} & =\frac{1}{2}\left(g_{\alpha \bar{\beta}} g_{\gamma \bar{\delta}}+g_{\gamma \bar{\beta}} g_{\alpha \bar{\delta}}\right), \\
\Phi_{\alpha \bar{\beta} \gamma \bar{\delta}} & =\frac{1}{2}\left(g_{\alpha \bar{\beta}} \eta_{\gamma} \eta_{\bar{\delta}}+g_{\gamma \bar{\beta}} \eta_{\alpha} \eta_{\bar{\delta}}+g_{\gamma \bar{\delta}} \eta_{\alpha} \eta_{\bar{\beta}}+g_{\alpha \bar{\delta}} \eta_{\gamma} \eta_{\bar{\beta}}\right), \\
\Psi_{\alpha \bar{\beta} \gamma \bar{\delta}} & =4 \eta_{\alpha} \eta_{\gamma} \eta_{\bar{\beta}} \eta_{\bar{\delta}} .
\end{aligned}
$$

From now on in this section we assume that the manifold $(M, g, J, D)$ is of quasiconstant holomorphic sectional curvatures, i.e. its curvature tensor $R$ satisfies the identity (3.1). Then the essential components of $\nabla R$ with respect to a complex basis $\left\{Z_{\alpha}\right\}, \alpha=$ $1, \ldots, n$ are the following:

$$
\begin{aligned}
& \nabla_{\alpha} R_{\beta \bar{\gamma} \delta \bar{\varepsilon}}= \\
& \frac{a_{\alpha}}{2}\left(g_{\beta \bar{\gamma}} g_{\delta \bar{\varepsilon}}+g_{\delta \bar{\gamma}} g_{\beta \bar{\varepsilon}}\right) \\
& +\frac{b_{\alpha}}{2}\left(g_{\beta \bar{\gamma}} \eta_{\delta} \eta_{\bar{\varepsilon}}+g_{\delta \bar{\gamma}} \eta_{\beta} \eta_{\bar{\varepsilon}}+g_{\delta \bar{\varepsilon}} \eta_{\beta} \eta_{\bar{\gamma}}+g_{\beta \bar{\varepsilon}} \eta_{\delta} \eta_{\bar{\gamma}}\right) \\
& +\frac{b}{2}\left\{g_{\beta \bar{\gamma}}\left(\nabla_{\alpha} \eta_{\delta} \cdot \eta_{\bar{\varepsilon}}+\eta_{\delta} \nabla_{\alpha} \eta_{\bar{\varepsilon}}\right)+g_{\delta \bar{\gamma}}\left(\nabla_{\alpha} \eta_{\beta} \cdot \eta_{\bar{\varepsilon}}+\eta_{\beta} \nabla_{\alpha} \eta_{\bar{\varepsilon}}\right)\right. \\
& \left.+g_{\delta \bar{\varepsilon}}\left(\nabla_{\alpha} \eta_{\beta} \cdot \eta_{\bar{\gamma}}+\eta_{\beta} \nabla_{\alpha} \eta_{\bar{\gamma}}\right)+g_{\beta \bar{\varepsilon}}\left(\nabla_{\alpha} \eta_{\delta} \cdot \eta_{\bar{\gamma}}+\eta_{\delta} \nabla_{\alpha} \eta_{\bar{\gamma}}\right)\right\} \\
& +4 c_{\alpha} \eta_{\beta} \eta_{\delta} \eta_{\bar{\gamma}} \eta_{\bar{\varepsilon}} \\
& +4 c\left(\nabla_{\alpha} \eta_{\beta} \cdot \eta_{\delta} \eta_{\bar{\gamma}} \eta_{\bar{\varepsilon}}+\eta_{\beta} \nabla_{\alpha} \eta_{\delta} \cdot \eta_{\bar{\gamma}} \eta_{\bar{\varepsilon}}+\eta_{\beta} \eta_{\delta} \nabla_{\alpha} \eta_{\bar{\gamma}} \cdot \eta_{\bar{\varepsilon}}+\eta_{\beta} \eta_{\delta} \eta_{\bar{\gamma}} \nabla_{\alpha} \eta_{\bar{\varepsilon}}\right) .
\end{aligned}
$$

Now we can find the integrability conditions following from the special form (3.1) of the curvature tensor $R$. 
Theorem 3.5. Let $(M, g, J, D)$ be a Kähler manifold with $\operatorname{dim} M=2 n \geq 6$ and curvature tensor $R$ satisfying the identity

$$
R=a \pi+b \Phi+c \Psi .
$$

Then the second Bianchi identity for the tensor $R$ is equivalent to the following conditions:

i) $d a=\frac{b d i v_{0} \xi}{2(n-1)} \eta+\frac{b d i v_{0} J \xi}{2(n-1)} \tilde{\eta}$;

ii) $d b=\frac{(b+4 c) d i v_{0} \xi}{n-1} \eta+\frac{(b+4 c) d i v_{0} J \xi}{n-1} \tilde{\eta}$;

iii) $b \nabla_{\lambda} \eta_{\mu}=0, \quad c \nabla_{\lambda} \eta_{\mu}=0$;

iv) $b\left\{\nabla_{\lambda} \eta_{\bar{\mu}}-\frac{d i v_{0} \xi}{2(n-1)} g_{\lambda \bar{\mu}}+\frac{d i v_{0} J \xi}{2(n-1)} \Omega_{\lambda \bar{\mu}}\right\}=0$;

$$
c\left\{\nabla_{\lambda} \eta_{\bar{\mu}}-\frac{d i v_{0} \xi}{2(n-1)} g_{\lambda \bar{\mu}}+\frac{d i v_{0} J \xi}{2(n-1)} \Omega_{\lambda \bar{\mu}}\right\}=0 ;
$$

v) $b \theta_{\lambda}=0, \quad b \theta_{\lambda}^{*}=0$;

vi) $c\left(\theta_{\lambda}+\theta_{\lambda}^{*}\right)=c_{\lambda}$

with respect to a special complex basis.

Proof. First we show that the second Bianchi identity for the curvature tensor $R=$ $a \pi+b \Phi+c \Psi$ implies the conditions i) - vi). The scheme of the proof is the following. The second Bianchi identity for the curvature tensor $R$ reduces to the equality

$$
\nabla_{\alpha} R_{\beta \bar{\gamma} \delta \bar{\varepsilon}}=\nabla_{\beta} R_{\alpha \bar{\gamma} \delta \bar{\varepsilon}}
$$

with respect to a complex basis $\left\{Z_{\alpha}\right\}, \alpha=1, \ldots, n$.

We replace $\nabla_{\alpha} R_{\beta \bar{\gamma} \delta \bar{\varepsilon}}$ and $\nabla_{\beta} R_{\alpha \bar{\gamma} \delta \bar{\varepsilon}}$ from (3.10) into (3.11). Further we use a special complex basis $\left\{Z_{0}, Z_{\lambda}\right\}, \lambda=1, \ldots n-1$, and substitute any of the vectors $Z_{\alpha}, \alpha=1, \ldots, n$ with $Z_{\lambda}, \lambda=1, \ldots, n-1$ or $Z_{0}$. Thus we obtain $2^{5}$ equalities which imply the conditions i) - vi) of the theorem. Here we give the main points of the proof:

1) $\nabla_{\lambda} R_{\mu \bar{\nu} \varkappa \bar{\sigma}}=\nabla_{\mu} R_{\lambda \bar{\nu} \varkappa \bar{\sigma}} \Longrightarrow$

$$
\begin{gathered}
\left(a_{\lambda} g_{\mu \bar{\nu}}-a_{\mu} g_{\lambda \bar{\nu}}\right) g_{\varkappa \bar{\sigma}}+\left(a_{\lambda} g_{\mu \bar{\sigma}}-a_{\mu} g_{\lambda \bar{\sigma}}\right) g_{\varkappa \bar{\nu}}=0 \Longrightarrow \\
a_{\lambda} g_{\mu \bar{\nu}}-a_{\mu} g_{\lambda \bar{\nu}}=0 \Longrightarrow(n-2) a_{\lambda}=0 \Longrightarrow a_{\lambda}=0 .
\end{gathered}
$$

2) $\nabla_{\lambda} R_{\mu \overline{0} 0 \bar{\sigma}}=\nabla_{\mu} R_{\lambda \overline{0} 0 \bar{\sigma}} \quad \Longrightarrow \quad(2 a+b)_{\lambda} g_{\mu \bar{\sigma}}-(2 a+b)_{\mu} g_{\lambda \bar{\sigma}}=0 \quad \Longrightarrow$

$$
(n-2)(2 a+b)_{\lambda}=0 \Longrightarrow b_{\lambda}=0 \text {. }
$$

3) $\nabla_{\lambda} R_{\mu \bar{\nu} \varkappa \overline{0}}=\nabla_{\mu} R_{\lambda \bar{\nu} \varkappa \overline{0}} \quad \Longrightarrow$

$$
\begin{gathered}
b\left(\nabla_{\lambda} \eta_{\mu} g_{\varkappa \bar{\nu}}-\nabla_{\mu} \eta_{\lambda} g_{\varkappa \bar{\nu}}+\nabla_{\lambda} \eta_{\varkappa} g_{\mu \bar{\nu}}-\nabla_{\mu} \eta_{\varkappa} g_{\lambda \bar{\nu}}\right)=0 \Longrightarrow \\
(n-2) b \nabla_{\mu} \eta_{\varkappa}=0 \Longrightarrow b \nabla_{\mu} \eta_{\varkappa}=0 .
\end{gathered}
$$

4) $\nabla_{0} R_{\mu \overline{0} \varkappa \overline{0}}=\nabla_{\mu} R_{0 \overline{0} \varkappa \overline{0}} \Longrightarrow(b+2 c) \nabla_{\mu} \eta_{\varkappa}=0 \quad \Longrightarrow \quad c \nabla_{\mu} \eta_{\varkappa}=0$. 
5) $\nabla_{0} R_{\mu \bar{\nu} \varkappa \bar{\sigma}}=\nabla_{\mu} R_{0 \bar{\nu} \varkappa \bar{\sigma}} \quad \Longrightarrow$

$$
\begin{gathered}
2 a_{0}\left(g_{\mu \bar{\nu}} g_{\varkappa \bar{\sigma}}+g_{\varkappa \bar{\nu}} g_{\mu \bar{\sigma}}\right)=b\left(\nabla_{\mu} \eta_{\bar{\sigma}} g_{\varkappa \bar{\nu}}+\nabla_{\mu} \eta_{\bar{\nu}} g_{\varkappa \bar{\sigma}}\right) \Longrightarrow \\
b \nabla_{\mu} \eta_{\bar{\nu}}=2 a_{0} g_{\mu \bar{\nu}}=\xi(a) g_{\mu \bar{\nu}}-J \xi(a) \Omega_{\mu \bar{\nu}} \Longrightarrow \\
\xi(a)=\frac{b d i v_{0} \xi}{2(n-1)}, \quad J \xi(a)=\frac{b d i v_{0} J \xi}{2(n-1)} ; \\
b\left\{\nabla_{\mu} \eta_{\bar{\nu}}-\frac{d i v_{0} \xi}{2(n-1)} g_{\mu \bar{\nu}}+\frac{d i v_{0} J \xi}{2(n-1)} \Omega_{\mu \bar{\nu}}\right\}=0 .
\end{gathered}
$$

6) $\nabla_{0} R_{\mu \overline{0} 0 \bar{\sigma}}=\nabla_{\mu} R_{0 \overline{0} 0 \bar{\sigma}} \quad \Longrightarrow$

$$
\begin{gathered}
(b+2 c) \nabla_{\mu} \eta_{\bar{\sigma}}=\frac{\xi(2 a+b)}{4} g_{\mu \bar{\sigma}}-\frac{J \xi(2 a+b)}{4} \Omega_{\mu \bar{\sigma}} \Longrightarrow \\
\xi(b)=(b+4 c) \frac{d i v_{0} \xi}{n-1}, \quad J \xi(b)=(b+4 c) \frac{d i v_{0} J \xi}{n-1} ; \\
c\left\{\nabla_{\mu} \eta_{\bar{\sigma}}-\frac{d i v_{0} \xi}{2(n-1)} g_{\mu \bar{\sigma}}+\frac{d i v_{0} J \xi}{2(n-1)} \Omega_{\mu \bar{\sigma}}\right\}=0 .
\end{gathered}
$$

In the next three points we use the identity $\left(\nabla_{X} \eta\right) \xi=0, \quad X \in \mathfrak{X} M$ which implies

$$
\nabla_{\alpha} \eta_{0}+\nabla_{\alpha} \eta_{\overline{0}}=0
$$

for any $Z_{\alpha}, \alpha=1, \ldots, n$.

7) $\nabla_{0} R_{\mu \bar{\nu} 0 \bar{\sigma}}=\nabla_{\mu} R_{0 \bar{\nu} 0 \bar{\sigma}} \quad \Longrightarrow \quad b\left\{g_{\mu \bar{\nu}} \nabla_{0} \eta_{\bar{\sigma}}+g_{\mu \bar{\sigma}} \nabla_{0} \eta_{\bar{\nu}}\right\}=0 \quad \Longrightarrow \quad b \nabla_{0} \eta_{\bar{\nu}}=0$.

Applying (3.7) we find

$$
b\left(\theta_{\bar{\nu}}-\theta_{\bar{\nu}}^{*}\right)=0 .
$$

8) $\nabla_{0} R_{\mu \overline{0} \varkappa \bar{\sigma}}=\nabla_{\mu} R_{0 \overline{0} \varkappa \bar{\sigma}} \quad \Longrightarrow \quad b\left\{g_{\varkappa \bar{\sigma}} \nabla_{0} \eta_{\mu}+g_{\mu \bar{\sigma}} \nabla_{0} \eta_{\varkappa}\right\}=0 \quad \Longrightarrow \quad b \nabla_{0} \eta_{\mu}=0$.

Applying (3.7) we obtain

$$
b\left(\theta_{\mu}+\theta_{\mu}^{*}\right)=0
$$

9) $\nabla_{0} R_{\mu \overline{0} 0 \overline{0}}=\nabla_{\mu} R_{0 \overline{0} \overline{0}} \quad \Longrightarrow \quad(b+2 c)\left(\theta_{\mu}+\theta_{\mu}^{*}\right)=2 c_{\mu} \quad \Longrightarrow$

$$
c\left(\theta_{\mu}+\theta_{\mu}^{*}\right)=c_{\mu} .
$$

Summarizing the results of 1) - 9) we obtain the equalities i) - vi) of the theorem.

Conversely, it is easy to check that the equalities i) - vi) imply the second Bianchi identity for $R$ (without assuming beforehand that it is true). Thus we obtained a complete system of integrability conditions (equivalent to the second Bianchi identity) for the curvature tensor $R=a \pi+b \Phi+c \Psi$.

QED

Corollary 3.6. Let $(M, g, J, D)$ be a Kähler manifolds satisfying the conditions in Theorem [3.5. If $\left(\operatorname{div}_{0} \xi(p), \operatorname{div}_{0} J \xi(p)\right) \neq(0,0)$ at every point $p \in M$, then the condition $b(p) \equiv 0$ implies $c(p) \equiv 0$. 
Here we study Kähler manifolds of quasi-constant holomorphic sectional curvatures, which have no points of constant holomorphic sectional curvatures, i.e.

$$
(b(p), c(p)) \neq(0,0), \quad p \in M .
$$

Under this condition we have as a consequence of Theorem 3.5

$$
\begin{aligned}
& d a=\frac{b d i v_{0} \xi}{2(n-1)} \eta+\frac{b d i v_{0} J \xi}{2(n-1)} \tilde{\eta}, \\
& d b=\frac{(b+4 c) d i v_{0} \xi}{n-1} \eta+\frac{(b+4 c) d i v_{0} J \xi}{n-1} \tilde{\eta}, \\
& \nabla_{\lambda} \eta_{\mu}=0, \quad \nabla_{\lambda} \eta_{\bar{\mu}}=\frac{d i v_{0} \xi}{2(n-1)} g_{\lambda \bar{\mu}}-\frac{d i v_{0} J \xi}{2(n-1)} \Omega_{\lambda \bar{\mu}}, \\
& b \theta_{\lambda}=0, \quad b \theta_{\lambda}^{*}=0, \quad c\left(\theta_{\lambda}+\theta_{\lambda}^{*}\right)=c_{\lambda} .
\end{aligned}
$$

There arise two geometric classes of Kähler manifolds of quasi-constant holomorphic sectional curvatures with respect to the distribution $D$.

The case: $\operatorname{div}_{0} \xi(p)=\operatorname{div}_{0} J \xi(p)=0$ at every point $p \in M$.

The equalities (3.18) reduce to the following

$$
\begin{aligned}
& d a=0, \quad d b=0, \\
& \nabla_{\lambda} \eta_{\mu}=0, \quad \nabla_{\lambda} \eta_{\bar{\mu}}=0, \\
& b \theta_{\lambda}=0, \quad b \theta_{\lambda}^{*}=0, \quad c\left(\theta_{\lambda}+\theta_{\lambda}^{*}\right)=c_{\lambda} .
\end{aligned}
$$

In this case the distribution $D$ is involutive according to Lemma 3.2 and the manifold $M$ is foliated by the integral submanifolds of $D$.

The case: $\left(\operatorname{div}_{0} \xi(p), \operatorname{div}_{0} J \xi(p)\right) \neq(0,0)$ at every point $p \in M$.

According to Corollary [3.6] if $b(p) \equiv 0, p \in M$, then $c(p) \equiv 0$, which contradicts to (3.17). Hence $b(p) \not \equiv 0$.

The aim of the present paper is to study the class of Kähler manifolds of quasi-constant holomorphic sectional curvatures satisfying the conditions

$$
\left(\operatorname{div}_{0} \xi(p), \operatorname{div}_{0} J \xi(p)\right) \neq(0,0), \quad b(p) \neq 0
$$

at every $p \in M$.

From (3.20) and (3.18) it follows that $d a(p) \neq 0, p \in M$. Then we can choose a new frame field $\left\{\xi^{\prime}, J \xi^{\prime}\right\}$ for $D^{\perp}$ such that $\eta^{\prime}=\frac{d a}{\|d a\|}$. This frame field satisfies the conditions $\operatorname{div}_{0} \xi^{\prime}(p) \neq 0, \operatorname{div}_{0} J \xi^{\prime}(p)=0$, at any point $p \in M$.

Now the conditions (3.18) reduce to the following:

$$
\begin{aligned}
& d a=\frac{b d i v_{0} \xi}{2(n-1)} \eta, \quad d b=\frac{(b+4 c) d i v_{0} \xi}{n-1} \eta \\
& \nabla_{\lambda} \eta_{\mu}=0, \quad \nabla_{\lambda} \eta_{\bar{\mu}}=\frac{d i v_{0} \xi}{2(n-1)} g_{\lambda \bar{\mu}} \\
& \theta_{\lambda}=0, \quad \theta_{\lambda}^{*}=0, \quad c_{\lambda}=0 .
\end{aligned}
$$




\section{Biconformal Transformations of Kähler metrics}

It is well known that any conformal transformation

$$
g^{\prime}=e^{2 u} g, \quad d u \neq 0
$$

of the metric $g$ in a Kähler manifold $(M, g, J)$ gives rise to a Hermitian manifold $\left(M, g^{\prime}, J\right)$ which is no more Kählerian .

The aim of our considerations in this section is to find the class of $J$-invariant distributions which admit biconformal changes of the given Kähler metric so that the new metrics continue being Kählerian. We obtain the group of the biconformal transformations and further apply it to the class of Kähler manifolds of quasi-constant holomorphic sectional curvatures.

First we deal with special distributions generated by real functions on the manifold.

Let $u$ be a real function of class $C^{\infty}$ on a Kähler manifold $(M, g, J)$ and $d u \neq 0$. Putting

$$
\xi=\frac{\operatorname{grad} u}{\|d u\|}, \quad J \xi=\frac{\operatorname{Jgrad} u}{\|d u\|}
$$

we obtain the $J$-invariant distribution $D$, where $D^{\perp}=\operatorname{span}\{\xi, J \xi\}$.

With respect to a special complex basis $\left\{Z_{0}, Z_{\lambda}\right\}, \lambda=1, \ldots, n-1$ we have $\nabla_{\lambda} u_{\bar{\mu}}=$ $\|d u\| \nabla_{\lambda} \eta_{\bar{\mu}}$. Since $\nabla_{\lambda} u_{\bar{\mu}}=\nabla_{\bar{\mu}} u_{\lambda}$, then $\nabla_{\lambda} \eta_{\bar{\mu}}=\nabla_{\bar{\mu}} \eta_{\lambda}$. The last equality implies that $\nabla_{\lambda} \eta^{\lambda}=\nabla_{\bar{\lambda}} \eta^{\bar{\lambda}}$ which is equivalent to $\operatorname{div}_{0} J \xi=0$.

We denote the relative Laplacian of $u$ with respect to $D$ by $\Delta_{0} u$ :

$$
\Delta_{0} u=2 g^{\lambda \bar{\mu}} \nabla_{\lambda} u_{\bar{\mu}}=\|d u\| d i v_{0} \xi
$$

Thus any real function $u$ with $d u \neq 0$ and $\Delta_{0} u \neq 0\left(d i v_{0} \xi \neq 0\right)$ generates a $J$-invariant distribution $D$, where $D^{\perp}=\operatorname{span}\{\xi, J \xi\}$, so that the frame field $\left\{\xi=\frac{\operatorname{grad} u}{\|u\|}, J \xi=\frac{J \operatorname{grad} u}{\|d u\|}\right\}$ is principal. What is more, the distribution

$$
\Delta(p)=\left\{X \in T_{p} M \mid \eta(X)=0\right\}, \quad p \in M
$$

associated with $\eta$ (perpendicular to $\xi$ ) is involutive.

The above considerations give motivation to study the geometric class of $J$-invariant distributions $D, D^{\perp}=\operatorname{span}\{\xi, J \xi\}$ satisfying the following conditions:

i) the distribution $\Delta$, perpendicular to $\xi$, is involutive;

ii) $\quad d i v_{0} \xi \neq 0$.

These conditions imply that $d i v_{0} J \xi=0$ and the frame field $\{\xi, J \xi\}$ is principal.

Since $\Delta$ is involutive, we can consider the class of proper functions of $\Delta$, i.e. all $C^{\infty}$ functions $q$ on $M$ satisfying the conditions (cf [5]):

$$
d q \neq 0, \quad d q=\|d q\| \eta=\xi(q) \eta
$$


For any proper function $q>0$ of $\Delta$ we determine the following transformation of the structure $(g, \eta)$ :

$$
\begin{aligned}
& g^{*}=g+(q-1)(\eta \otimes \eta+\tilde{\eta} \otimes \tilde{\eta}), \quad q>0 ; \\
& \eta^{*}=\sqrt{q} \eta, \quad \xi^{*}=\frac{1}{\sqrt{q}} \xi .
\end{aligned}
$$

The condition $q>0$ implies $g^{*}$ is a Hermitian metric. We shall call the transformations (4.3) complex dilatational transformations of the structure $(g, \eta)$ (cf dilatational transformations in a Riemannian manifold $(M, g, \xi)[5])$. We recall that the frame field $\{\xi, J \xi\}$ spanning $D^{\perp}$ is principal and then the transformation (4.3) is an object in the geometry of $U(n-1) \times U(1)$.

In general, the manifold $\left(M, g^{*}, J, D\right)$ is a Hermitian one with metric $g^{*}$ and Kähler form $\Omega^{*}(X, Y)=g^{*}(J X, Y), \quad X, Y \in \mathfrak{X} M$.

Any complex dilatational transformation (4.3) is given by the following equalities:

$$
g_{\beta \bar{\gamma}}^{*}=g_{\beta \bar{\gamma}}+2(q-1) \eta_{\beta} \eta_{\bar{\gamma}} ; \quad \eta_{\alpha}^{*}=\sqrt{q} \eta_{\alpha}
$$

with respect to a complex basis $\left\{Z_{\alpha}\right\}, \alpha=1, \ldots, n$.

Then the Kähler forms $\Omega$ and $\Omega^{*}$ of both structures $(g, J)$ and $\left(g^{*}, J\right)$ are related as follows

$$
\Omega_{\beta \bar{\gamma}}^{*}=\Omega_{\beta \bar{\gamma}}+2 i(q-1) \eta_{\beta} \eta_{\bar{\gamma}} .
$$

First we shall recall some notions and facts about the Hermitian structures $\left(g^{*}, J\right)$.

The Lee form $\omega^{*}$ of the structure $\left(g^{*}, J\right)$ is determined by

$$
\omega_{\alpha}^{*}=-\frac{i}{n-1} d \Omega_{\alpha \beta \bar{\gamma}}^{*} g^{* \beta \bar{\gamma}} .
$$

A Hermitian manifold $\left(M, g^{*}, J\right)$ with $\operatorname{dim} M=2 n \geq 6$ is locally conformal Kähler (a $W_{4}$-manifold in the classification scheme of almost Hermitian manifolds [4]) if and only if

$$
d \Omega^{*}=\omega^{*} \wedge \Omega^{*}
$$

It is well known that (4.7) implies $\omega^{*}$ is closed in $\operatorname{dim} M \geq 6$.

If $u$ is a local solution of the equation $2 d u=-\omega^{*}$, then the metric $e^{2 u} g^{*}$ is Kählerian.

Now we can find the conditions for a $J$-invariant distribution $D$ of type (4.1), under which all metrics $g^{*}$ given by (4.3) are locally conformal Kählerian.

Lemma 4.1. Let $(M, g, J, D) \quad(\operatorname{dim} M=2 n \geq 6)$ be a Kähler manifold with $J$-invariant distributions $D, D^{\perp}=\operatorname{span}\{\xi, J \xi\}$ of type (4.1). Then every structure $\left(g^{*}, J\right)$, where $g^{*}$ is a metric obtained from $g$ by a complex dilatational transformation (4.3), is locally conformal Kählerian if and only if the distributions $D, D^{\perp}$ satisfy the following conditions:

i) $\theta_{\mu}+\theta_{\mu}^{*}=0$

ii) $\nabla_{\lambda} \eta_{\bar{\mu}}=\frac{d i v_{0} \xi}{2(n-1)} g_{\lambda \bar{\mu}}$

with respect to a special complex basis $\left\{Z_{0}, Z_{\lambda}\right\}, \lambda=1, \ldots, n-1$. 
Proof. Let $g^{*}$ be any metric given by (4.3).

We shall use complex bases and special complex bases taking into account the convention about the indices $\alpha, \beta, \gamma$ and $\lambda, \mu, \nu$, respectively.

Differentiating (4.5) with respect to the Levi-Civita connection $\nabla$ of the metric $g$ and taking into account (4.2) we find

$$
\begin{aligned}
d \Omega_{\alpha \beta \bar{\gamma}}^{*} & =\nabla_{\alpha} \Omega_{\beta \bar{\gamma}}^{*}-\nabla_{\beta} \Omega_{\alpha \bar{\gamma}}^{*} \\
& =2 i(q-1)\left(d \eta_{\alpha \beta} \eta_{\bar{\gamma}}+\eta_{\beta} \nabla_{\alpha} \eta_{\bar{\gamma}}-\eta_{\alpha} \nabla_{\beta} \eta_{\bar{\gamma}}\right) .
\end{aligned}
$$

The components $g^{* \beta \bar{\gamma}}$ of the inverse matrix of $\left(g_{\beta \bar{\gamma}}^{*}\right)$ can be found from (4.4). The corresponding calculations give

$$
g^{* \beta \bar{\gamma}}=g^{\beta \bar{\gamma}}-\frac{2(q-1)}{q} \eta^{\beta} \eta^{\bar{\gamma}} .
$$

Replacing $d \Omega_{\alpha \beta \bar{\gamma}}^{*}$ from (4.8) and $g^{* \beta \bar{\gamma}}$ from (4.9) into (4.6) we get

$$
\omega_{\alpha}^{*}=-\frac{(q-1) d i v_{0} \xi}{n-1} \eta_{\alpha}-\frac{q-1}{(n-1) q}\left(\theta_{\alpha}+\theta_{\alpha}^{*}\right) .
$$

Consequently

$$
\omega_{\lambda}^{*}=-\frac{q-1}{(n-1) q}\left(\theta_{\lambda}+\theta_{\lambda}^{*}\right) .
$$

If the structure $\left(g^{*}, J\right)$ is locally conformal Kählerian, then

$$
d \Omega_{\alpha \beta \bar{\gamma}}^{*}=\omega_{\alpha}^{*} \Omega_{\beta \bar{\gamma}}^{*}-\omega_{\beta}^{*} \Omega_{\alpha \bar{\gamma}}^{*}=i\left(\omega_{\alpha}^{*} g_{\beta \bar{\gamma}}-\omega_{\beta}^{*} g_{\alpha \bar{\gamma}}\right) .
$$

Because of (4.8) the equality (4.12) takes the form

$$
2(q-1)\left(d \eta_{\alpha \beta} \eta_{\bar{\gamma}}+\eta_{\beta} \nabla_{\alpha} \eta_{\bar{\gamma}}-\eta_{\alpha} \nabla_{\beta} \eta_{\bar{\gamma}}\right)=\omega_{\alpha}^{*} g_{\beta \bar{\gamma}}^{*}-\omega_{\beta}^{*} g_{\alpha \bar{\gamma}}^{*} .
$$

Replacing $\alpha, \beta, \gamma$ into (4.13) with $\lambda, \mu, \nu$, respectively, we obtain

$$
\omega_{\lambda}^{*} g_{\mu \bar{\nu}}^{*}-\omega_{\mu}^{*} g_{\lambda \bar{\nu}}^{*}=0 \Longrightarrow(n-2) \omega_{\lambda}^{*}=0 \quad \Longrightarrow \quad \omega_{\lambda}^{*}=0 .
$$

Then it follows from (4.11) that

$$
(q-1)\left(\theta_{\lambda}+\theta_{\lambda}^{*}\right)=0 .
$$

Since the last equality is fulfilled for every proper function $q$ from $(4.3)$, then $\theta_{\lambda}+\theta_{\lambda}^{*}=0$, which is $\mathrm{i}$ ).

Further we replace $\alpha, \beta, \gamma$ into (4.13) with $0, \mu, \nu$, respectively. Using (3.4) and (4.10) we get

$$
-(q-1) \nabla_{\mu} \eta_{\bar{\nu}}=\omega_{0}^{*} g_{\mu \bar{\nu}}^{*}=\omega_{0}^{*} g_{\mu \bar{\nu}}=-\frac{(q-1) d i v_{0} \xi}{2(n-1)} g_{\mu \bar{\nu}}
$$

which implies the condition ii).

Conversely, it is an immediate verification that the conditions i) and ii) in view of (3.4) and (3.7) make (4.13) an identity.

Hence, every structure $\left(g^{*}, J\right)$ is locally conformal Kählerian. 
Remark 4.2. The formula (4.10) and Lemma 3.3 imply that the following conditions are equivalent:

i) $\theta_{\mu}+\theta_{\mu}^{*}=0$;

ii) the distribution $D^{\perp}$ is involutive;

iii) every Lee form $\omega^{*}$ is collinear with the structural 1-form $\eta$.

Remark 4.3. The condition ii) in the above lemma in view of the properties of the $J$ invariant distributions $D, D^{\perp}$ is equivalent to

$$
d \tilde{\eta}\left(x_{0}, y_{0}\right)=\frac{d i v_{0} \xi}{n-1} \Omega\left(x_{0}, y_{0}\right), \quad x_{0}, y_{0} \in D .
$$

Since the function $\frac{d i v_{0} \xi}{n-1}=-\frac{\delta_{0} \eta}{n-1}$ occurs quite frequently in what follows, for the sake of brevity we shall use the denotation

$$
k=\frac{d i v_{0} \xi}{n-1} .
$$

In view of Lemma 4.1 we introduce the following notion

Definition 4.4. Let $(M, g, J, D)(\operatorname{dim} M=2 n \geq 6)$ be a Kähler manifold with $J$ invariant distributions $D, D^{\perp}=\operatorname{span}\{\xi, J \xi\}$. The distribution $D$ is said to be a $B$ distribution if the following conditions hold good:

i) the distribution $\Delta$ orthogonal to $\xi$ is involutive;

ii) the distribution $D^{\perp}$ is involutive;

iii) $\quad d \tilde{\eta}_{\mid D}=k \Omega_{\mid D}, \quad k \neq 0$.

It follows from the above definition that

$$
\begin{aligned}
& \nabla_{\lambda} \eta_{\mu}=\nabla_{\mu} \eta_{\lambda}, \quad \nabla_{\lambda} \eta_{\bar{\mu}}=\frac{k}{2} g_{\lambda \bar{\mu}}, \\
& \frac{d i v_{0} \xi}{n-1}=k \neq 0, \quad d i v_{0} J \xi=0 .
\end{aligned}
$$

Thus we have:

The geometry of a B-distribution in a Kähler manifold is determined by:

the symmetric tensor $\nabla_{\lambda} \eta_{\mu}$;

the 1 -form $\theta$;

the functions $k, p$ and $p^{*}$.

Now we can introduce transformations of the tensors $g, \eta$ changing the Kähler structure $(g, J)$ into a Kähler structure $\left(g^{\prime}, J\right)$.

Let $(M, g, J, D)(\operatorname{dim} M=2 n \geq 6)$ be a Kähler manifold with $B$-distribution $D\left(D^{\perp}=\right.$ $\operatorname{span}\{\xi, J \xi\})$. If

$$
g^{*}=g+(q-1)(\eta \otimes \eta+\tilde{\eta} \otimes \tilde{\eta}), \quad q>0, \quad d q=\xi(q) \eta
$$


is a complex dilatational change of the metric $g$, then $\left(g^{*}, J\right)$ is a locally conformal Kähler structure whose Lee form $\omega^{*}$ in view of (4.10) is given by

$$
\omega^{*}=-(q-1) k \eta \text {. }
$$

If $u$ is a local solution of the equation

$$
2 d u=-\omega^{*}=k(q-1) \eta
$$

then $u$ is a proper function of the distribution $\Delta$ and $2 \xi(u)=k(q-1)$. Putting

$$
g^{\prime}=e^{2 u} g^{*}=e^{2 u}\{g+(q-1)(\eta \otimes \eta+\tilde{\eta} \otimes \tilde{\eta})\}
$$

we obtain $g^{\prime}$ is a Kähler metric.

It is clear that the metric $g^{\prime}$ is determined by the proper function $q$ up to a constant factor.

Since $q>0$ we set $q=e^{2 v}$ and obtain another form of the metric $g^{\prime}$ :

$$
g^{\prime}=e^{2 u}\left\{g+\left(e^{2 v}-1\right)(\eta \otimes \eta+\tilde{\eta} \otimes \tilde{\eta})\right\} .
$$

Definition 4.5. Let $(M, g, J, D)(\operatorname{dim} M=2 n \geq 6)$ be a Kähler manifold with $B$ distribution $D$. The transformation

$$
\begin{aligned}
& g^{\prime}=e^{2 u}\{g-(\eta \otimes \eta+\tilde{\eta} \otimes \tilde{\eta})\}+e^{2(u+v)}(\eta \otimes \eta+\tilde{\eta} \otimes \tilde{\eta}) ; \\
& \eta^{\prime}=e^{u+v} \eta, \quad \xi^{\prime}=e^{-(u+v)} \xi ; \\
& d v=\xi(v) \eta \neq 0, \quad 2 d u=k\left(e^{2 v}-1\right) \eta .
\end{aligned}
$$

is said to be a biconformal transformation of the structure $(g, \eta)$.

From the above definition it follows that $\left(M, g^{\prime}, J, D\right)$ is a Kähler manifold with the same distributions $D, D^{\perp}$ and $\Delta$. By a given proper function $v$ the metric $g^{\prime}$ is determined up to a constant factor (homothety).

Proposition 4.6. Let $(M, g, J, D)(\operatorname{dim} M=2 n \geq 6)$ be a Kähler manifold with Bdistribution $D$ and $\left(g^{\prime}, \eta^{\prime}\right)$ be any structure obtained from the structure $(g, \eta)$ by a biconformal transformation. Then $D$ continues being a $B$-distribution with respect to the new structure $\left(g^{\prime}, \eta^{\prime}\right)$.

Proof. Let the structure $\left(g^{\prime}, \eta^{\prime}\right)$ be given by (4.16). Since the distributions $\Delta$ and $D^{\perp}$ do not change, then the first two conditions of Definition 4.4 are fulfilled.

To prove the third condition of Definition 4.4 we differentiate the equality $\tilde{\eta}^{\prime}=e^{u+v} \tilde{\eta}$ and taking into account (4.16) we get

$$
d \tilde{\eta}_{\mid D}^{\prime}=e^{u+v} d \tilde{\eta}_{\mid D}=e^{u+v} k \Omega_{\mid D}=e^{v-u} k \Omega_{\mid D}^{\prime} .
$$

Hence

$$
d \tilde{\eta}_{\mid D}^{\prime}=k^{\prime} \Omega_{\mid D}^{\prime}
$$

where

$$
k^{\prime}=e^{v-u} k .
$$


KÄHLER MANIFOLDS OF QUASI-CONSTANT HOLOMORPHIC CURVATURES

Proposition 4.7. Let $(M, g, J, D)(\operatorname{dim} M=2 n \geq 6)$ be a Kähler manifold with Bdistribution $D$. Then the biconformal transformations of the structure $(g, \eta)$ form a group.

Proof. I. Let $v$ be a proper function of the distribution $\Delta$, i.e. $d v=\xi(v) \eta \neq 0$ and

$$
\begin{gathered}
g^{\prime}=e^{2 u}\{g-(\eta \otimes \eta+\tilde{\eta} \otimes \tilde{\eta})\}+e^{2(u+v)}(\eta \otimes \eta+\tilde{\eta} \otimes \tilde{\eta}), \\
\eta^{\prime}=e^{u+v} \eta, \quad d u=\frac{k\left(e^{2 v}-1\right)}{2} \eta .
\end{gathered}
$$

By direct computations taking into account (4.17) we find the inverse transformation

$$
\begin{gathered}
g=e^{-2 u}\left\{g^{\prime}-\left(\eta^{\prime} \otimes \eta^{\prime}+\tilde{\eta}^{\prime} \otimes \tilde{\eta}^{\prime}\right)\right\}+e^{-2(u+v)}\left(\eta^{\prime} \otimes \eta^{\prime}+\tilde{\eta}^{\prime} \otimes \tilde{\eta}^{\prime}\right), \\
\eta=e^{-(u+v)} \eta^{\prime}, \quad d(-u)=\frac{k^{\prime}\left(e^{-2 v}-1\right)}{2} \eta^{\prime} .
\end{gathered}
$$

II. Let $v^{\prime}$ and $v^{\prime \prime}$ be proper functions of the distribution $\Delta$ generating the following biconformal transformations:

$$
\begin{gathered}
g^{\prime}=e^{2 u^{\prime}}\{g-(\eta \otimes \eta+\tilde{\eta} \otimes \tilde{\eta})\}+e^{2\left(u^{\prime}+v^{\prime}\right)}(\eta \otimes \eta+\tilde{\eta} \otimes \tilde{\eta}), \\
\eta^{\prime}=e^{u^{\prime}+v^{\prime}} \eta, \quad d u^{\prime}=\frac{k\left(e^{2 v^{\prime}}-1\right)}{2} \eta ; \\
g^{\prime \prime}=e^{2 u^{\prime \prime}}\left\{g^{\prime}-\left(\eta^{\prime} \otimes \eta^{\prime}+\tilde{\eta}^{\prime} \otimes \tilde{\eta}^{\prime}\right)\right\}+e^{2\left(u^{\prime \prime}+v^{\prime \prime}\right)}\left(\eta^{\prime} \otimes \eta^{\prime}+\tilde{\eta}^{\prime} \otimes \tilde{\eta}^{\prime}\right), \\
\eta^{\prime \prime}=e^{u^{\prime \prime}+v^{\prime \prime}} \eta^{\prime}, \quad d u^{\prime \prime}=\frac{k^{\prime}\left(e^{2 v^{\prime \prime}}-1\right)}{2} \eta^{\prime} .
\end{gathered}
$$

It is easy to check that

$$
\begin{gathered}
g^{\prime \prime}=e^{2\left(u^{\prime}+u^{\prime \prime}\right)}\{g-(\eta \otimes \eta+\tilde{\eta} \otimes \tilde{\eta})\}+e^{2\left(u^{\prime}+u^{\prime \prime}+v^{\prime}+v^{\prime \prime}\right)}(\eta \otimes \eta+\tilde{\eta} \otimes \tilde{\eta}), \\
\eta^{\prime \prime}=e^{u^{\prime}+u^{\prime \prime}+v^{\prime}+v^{\prime \prime}} \eta, \quad d\left(u^{\prime}+u^{\prime \prime}\right)=\frac{k\left(e^{2\left(v^{\prime}+v^{\prime \prime}\right)}-1\right)}{2} \eta .
\end{gathered}
$$

Taking into account I and II we conclude that the biconformal transformations (4.16) form a group, which is generated by the additive group of the real $C^{\infty}$-functions on $M$. QED

\section{TENSOR INVARIANTS OF THE BICONFORMAL GROUP OF TRANSFORMATIONS}

Let $(M, g, J, D)$ be a Kähler manifold with $B$-distribution $D$. In order to find tensor invariants of the group of biconformal transformations we shall further specialize the distribution $D$.

Taking into account that the $J$-invariant distribution of any Kähler $Q C H$-manifold of the class under consideration is a $B$-distribution satisfying the conditions $\nabla_{\lambda} \eta_{\mu}=0$ and $\theta=\theta^{*}=0$ we introduce the following type of $J$-invariant distributions: 
Definition 5.1. Let $(M, g, J, D)$ be a Kähler manifold with $J$-invariant distribution $D$ of codimension two. The distribution $D$ is said to be a $B_{0}$-distribution if it satisfies the following conditions:

1) $D$ is a $B$-distribution;

2) $\left(\nabla_{x_{0}} \eta\right)\left(y_{0}\right)-\left(\nabla_{J x_{0}} \eta\right)\left(J y_{0}\right)=0, \quad x_{0}, y_{0} \in D$;

3) $\theta=0$.

From the above definition it follows immediately that $\theta^{*}=0$.

Under the assumptions (5.1) we shall find additional conditions for the basic functions $p$ and $p^{*}$, introduced by (3.5), and $k=\frac{d i v_{0} \xi}{n-1}$.

First we precise the form of the tensor $\nabla \eta$.

Lemma 5.2. Let $(M, g, J, D)$ be a Kähler manifold with $B_{0}$-distribution $D$. Then

$$
\begin{aligned}
\left(\nabla_{X} \eta\right) Y= & \frac{k}{2}\{g(X, Y)-\eta(X) \eta(Y)-\tilde{\eta}(X) \tilde{\eta}(Y)\} \\
& +p \eta(X) \tilde{\eta}(Y)-p^{*} \tilde{\eta}(X) \tilde{\eta}(Y) ; \quad X, Y \in \mathfrak{X} M .
\end{aligned}
$$

Proof. We consider a special complex basis $\left\{Z_{0}, Z_{\lambda}\right\}, \lambda=1, \ldots, n-1$. Then the assertion of the lemma is equivalent to the equalities:

$$
\begin{aligned}
& \nabla_{\lambda} \eta_{\mu}=0, \quad \nabla_{\lambda} \eta_{\bar{\mu}}=\frac{k}{2} g_{\lambda \bar{\mu}} \\
& \nabla_{0} \eta_{\mu}=0, \quad \nabla_{\overline{0}} \eta_{\mu}=0 ; \\
& \nabla_{\lambda} \eta_{0}=0, \quad \nabla_{\lambda} \eta_{\overline{0}}=0 ; \\
& \nabla_{0} \eta_{0}=-\nabla_{0} \eta_{\overline{0}}=\frac{1}{4}\left(p^{*}-i p\right) .
\end{aligned}
$$

The first two equalities of (5.2) are given by the conditions 1) and 2) of (5.1). The last two equalities of (5.2) follow from the formulas (3.7) and the condition 3).

Since the distribution $\Delta$ orthogonal to $\xi$ is involutive, then $d \eta\left(x_{0}, J \xi\right)=0$ for any $x_{0} \in D$. The last equality and the condition $\theta^{*}=0$ imply $g\left(\nabla_{x_{0}} \xi, J \xi\right)=0, x_{0} \in D$. From here we obtain the first two equalities of (5.3). The remaining equalities of (5.3) are given by the formulas (3.8).

QED

In the next lemma we obtain integrability conditions concerning the functions $k, p$ and $p^{*}$ as a consequence of the conditions $\nabla_{\lambda} \eta_{\mu}=0$ and $\theta=\theta^{*}=0$.

Lemma 5.3. Let $(M, g, J, D)(\operatorname{dim} M=2 n \geq 6)$ be a Kähler manifold with $B_{0}$-distribution. Then the following equalities

$$
p=0, \quad d k=-k\left(k+p^{*}\right) \eta, \quad d p^{*}=\xi\left(p^{*}\right) \eta
$$

hold good.

Proof. Applying Lemma 5.2 we obtain

$$
d \eta=p \eta \wedge \tilde{\eta}
$$




$$
d \tilde{\eta}=k \Omega-\left(k+p^{*}\right) \eta \wedge \tilde{\eta} .
$$

The exterior derivation of (5.4) because of (5.5) gives

$$
k p\left(\eta_{\alpha} g_{\beta \bar{\gamma}}-\eta_{\beta} g_{\alpha \bar{\gamma}}\right)=2\left(p_{\alpha} \eta_{\beta}-p_{\beta} \eta_{\alpha}\right) \eta_{\bar{\gamma}}
$$

with respect to a complex basis $\left\{Z_{\alpha}\right\}, \alpha=1, \ldots, n$.

Passing on a special complex basis $\left\{Z_{0}, Z_{\lambda}\right\}, \lambda=1, \ldots, n-1$ we substitute $\alpha=0, \beta=$ $\mu, \gamma=\nu$ in the above equality and find $k p=0$. Since $k \neq 0$, then

$$
p=0 \text {. }
$$

A similar operation applied to (5.5) gives as a result the equality

$$
\begin{aligned}
& \left\{k_{\alpha}+k\left(k+p^{*}\right) \eta_{\alpha}\right\} g_{\beta \bar{\gamma}}-\left\{k_{\beta}+k\left(k+p^{*}\right) \eta_{\beta}\right\} g_{\alpha \bar{\gamma}} \\
& -2\left\{\left(k+p^{*}\right)_{\alpha} \eta_{\beta}-\left(k+p^{*}\right)_{\beta} \eta_{\alpha}\right\} \eta_{\bar{\gamma}}=0 .
\end{aligned}
$$

Proceeding to a special complex basis in (5.6) we substitute $\alpha=\lambda, \beta=\mu, \gamma=\nu$ and get

$$
k_{\lambda} g_{\mu \bar{\nu}}-k_{\mu} g_{\lambda \bar{\nu}}=0 \quad \Longrightarrow \quad(n-2) k_{\lambda}=0 \quad \Longrightarrow \quad k_{\lambda}=0
$$

The substitution $\alpha=0, \beta=\mu, \gamma=\nu$ into (5.6) gives as a consequence

$$
\left\{k_{0}+\frac{1}{2} k\left(k+p^{*}\right)\right\} g_{\mu \bar{\nu}}=0
$$

which implies $J \xi(k)=0, \quad \xi(k)=-k\left(k+p^{*}\right)$.

Thus we obtained the equalities

$$
k_{\lambda}=0, \quad J \xi(k)=0, \quad \xi(k)=-k\left(k+p^{*}\right),
$$

which are equivalent to

$$
d k=\xi(k) \eta=-k\left(k+p^{*}\right) \eta
$$

The exterior derivation in the last equality gives $d p^{*} \wedge \eta=0$, i.e.

$$
d p^{*}=\xi\left(p^{*}\right) \eta \text {. }
$$

Now, taking into account Lemma 5.3, we have from Lemma 5.2

$$
\nabla \eta=\frac{k}{2}(g-\eta \otimes \eta-\tilde{\eta} \otimes \tilde{\eta})-p^{*} \tilde{\eta} \otimes \tilde{\eta}
$$

where the function $p^{*}$ in view of (5.7) satisfies the relation

$$
p^{*}=-\frac{\xi(k)+k^{2}}{k} .
$$

The equality (5.8) is equivalent to the equalities

$$
\begin{gathered}
\nabla_{\alpha} \eta_{\beta}=p^{*} \eta_{\alpha} \eta_{\beta}, \\
\nabla_{\alpha} \eta_{\bar{\beta}}=\frac{k}{2} g_{\alpha \bar{\beta}}-\left(k+p^{*}\right) \eta_{\alpha} \eta_{\bar{\beta}}
\end{gathered}
$$

with respect to a complex basis $\left\{Z_{\alpha}\right\}, \alpha=1, \ldots, n$. 
Lemma 5.4. Let $(M, g, J, D)(\operatorname{dim} M=2 n \geq 6)$ be a Kähler manifold with $B_{0}$-distribution D. Then the following equalities hold good:

$$
\begin{gathered}
\sigma=\frac{1}{2 k} \xi\left(k^{2}+2 k p^{*}\right)+\frac{n+1}{2}\left(k^{2}+2 k p^{*}\right) ; \\
\varkappa=\frac{1}{2 k} \xi\left(k^{2}+2 k p^{*}\right)+k^{2}+2 k p^{*} ; \\
R(X, Y) \xi=4 \frac{\sigma-\varkappa}{n-1}\{\Phi(X, Y) \xi-\Psi(X, Y) \xi\}+\varkappa \Psi(X, Y) \xi .
\end{gathered}
$$

Proof. Writing the equality (5.8) in the form

$$
\nabla_{X} \xi=\frac{k}{2}\{X-\eta(X) \xi-\tilde{\eta}(X) J \xi\}-p^{*} \tilde{\eta}(X) J \xi, \quad X \in \mathfrak{X} M,
$$

we get in a straightforward way

$$
\begin{aligned}
R(X, Y) \xi= & -\frac{1}{4}\left(k^{2}+2 k p^{*}\right)\{\eta(X) Y-\eta(Y) X \\
& -\tilde{\eta}(X) J Y+\tilde{\eta}(Y) J X+2 g(J X, Y) J \xi\} \\
& -\frac{1}{2 k} \xi\left(k^{2}+2 k p^{*}\right)\{\eta(X) \tilde{\eta}(Y)-\eta(Y) \tilde{\eta}(X)\} J \xi,
\end{aligned}
$$

$X, Y \in \mathfrak{X} M$.

Taking a trace in (5.15) we find

$$
\rho(X, \xi)=\left\{\frac{1}{2 k} \xi\left(k^{2}+2 k p^{*}\right)+\frac{n+1}{2}\left(k^{2}+2 k p^{*}\right)\right\} \eta(X), \quad X \in \mathfrak{X} M,
$$

which implies (5.12).

Further we replace $X=J \xi, Y=\xi$ into (5.15) and find (5.13).

Taking into account (5.15), (2.5), (2.6), (5.12) and (5.13) we obtain (5.14).

QED

Remark 5.5. Let $D$ be a $B_{0}$-distribution. Then the equality (5.16) means that $\xi$ is an eigen vector field for the Ricci operator $\rho$.

Further the equality (5.14) implies that the manifold under consideration is of pointwise constant mixed sectional curvatures

$$
R\left(x_{0}, \xi, \xi, x_{0}\right)=\frac{\sigma-\varkappa}{2(n-1)}=\frac{1}{4}\left(k^{2}+2 k p^{*}\right), \quad x_{0} \in D,\left\|x_{0}\right\|=1 .
$$

What is more, the equalities (5.17), (5.13) and (5.9) express that the function $k$ completely determines the mixed sectional curvatures and the vertical sectional curvature of the manifold.

The next assertion justifies the introduction of the class of $B_{0}$-distributions.

Proposition 5.6. Let $(M, g, J, D)(\operatorname{dim} M=2 n \geq 6)$ be a Kähler manifold with $B_{0}$ distribution $D$ and $D^{\perp}=\operatorname{span}\{\xi, J \xi\}$. If $\left(g^{\prime}, \eta^{\prime}\right)$ is any structure obtained from the given structure $(g, \eta)$ by a biconformal transformation, then $D$ is also a $B_{0}$-distribution with respect to the new structure $\left(g^{\prime}, \eta^{\prime}\right)$. 
Proof. Let

$$
\begin{gathered}
g^{\prime}=e^{2 u}\left\{g+\left(e^{2 v}-1\right)(\eta \otimes \eta+\tilde{\eta} \otimes \tilde{\eta})\right\}, \quad \eta^{\prime}=e^{u+v} \eta \\
d v=\xi(v) \eta, \quad d u=\frac{k\left(e^{2 v}-1\right)}{2} \eta
\end{gathered}
$$

be a biconformal change of the structure $(g, \eta)$ and $\nabla^{\prime}$ be the Levi-Civita connection of the metric $g^{\prime}$.

According to Proposition 4.6 the distribution $D$ is a $B$-distribution with respect to the structure $\left(g^{\prime}, \eta^{\prime}\right)$. It remains to prove that $\theta^{\prime}=0$ and $\left(\nabla_{x_{0}}^{\prime} \eta^{\prime}\right)\left(y_{0}\right)-\left(\nabla_{J x_{0}}^{\prime} \eta^{\prime}\right)\left(J y_{0}\right)=$ $0, \quad x_{0}, y_{0} \in D$.

By direct computations we find the relation between $\nabla^{\prime}$ and $\nabla$ :

$$
\begin{aligned}
\nabla_{X}^{\prime} Y= & \nabla_{X} Y+\xi(u)\{\eta(X) Y+\eta(Y) X+\tilde{\eta}(X) J Y+\tilde{\eta}(Y) J X\} \\
& +\xi(v-u)\{(\eta(X) \eta(Y)-\tilde{\eta}(X) \tilde{\eta}(Y)) \xi \\
& +(\eta(X) \tilde{\eta}(Y)+\tilde{\eta}(X) \eta(Y)) J \xi\} ; \quad X, Y \in \mathfrak{X} M .
\end{aligned}
$$

Then we find the components of $\nabla^{\prime} \eta^{\prime}$ with respect to a complex basis:

$$
\begin{gathered}
\nabla_{\alpha}^{\prime} \eta_{\beta}^{\prime}=e^{-(u+v)}\left\{p^{*}-\xi(u+v)\right\} \eta_{\alpha}^{\prime} \eta_{\beta}^{\prime}, \\
\nabla_{\alpha}^{\prime} \eta_{\bar{\beta}}^{\prime}=e^{v-u} \frac{k}{2}\left(g_{\alpha \bar{\beta}}^{\prime}-2 \eta_{\alpha}^{\prime} \eta_{\bar{\beta}}^{\prime}\right)-e^{-(u+v)}\left\{p^{*}-\xi(u+v)\right\} \eta_{\alpha}^{\prime} \eta_{\bar{\beta}}^{\prime} .
\end{gathered}
$$

Now let $\left\{Z_{\lambda}^{\prime}, Z_{0}^{\prime}\right\}, \lambda=1, \ldots, n-1$ be a special complex basis with respect to the structure $\left(g^{\prime}, \eta^{\prime}\right)$. Taking into account $(5.20),(5.21)$ and (3.7) we get

$$
\nabla_{\lambda}^{\prime} \eta_{\mu}^{\prime}=0, \quad \nabla_{\lambda}^{\prime} \eta_{0}^{\prime}=\frac{\theta_{\lambda}^{\prime}+\theta_{\lambda}^{\prime *}}{2}=0, \quad \nabla_{\lambda}^{\prime} \eta_{\overline{0}}^{\prime}=\frac{\theta_{\lambda}^{\prime}-\theta_{\lambda}^{\prime *}}{2}=0
$$

which implies the assertion.

The equality (5.21) implies the change (4.17) of the function $k$ and the change of the function $p^{*}$ :

$$
p^{* \prime}=e^{-(u+v)}\left\{p^{*}-\xi(u+v)\right\} .
$$

Now we can prove the main theorem in this section.

Theorem 5.7. Let $(M, g, J, D)(\operatorname{dim} M=2 n \geq 6)$ be a Kähler manifold with $B_{0^{-}}$ distribution $D$ and $D^{\perp}=\operatorname{span}\{\xi, J \xi\}$. Then the tensor of type (1.3)

$$
Q C(R)=R-a \pi-b \Phi-c \Psi
$$

is a biconformal invariant.

Proof. Let $\left(g^{\prime}, \eta^{\prime}\right)$ be a structure obtained from the given structure $(g, \eta)$ by the biconformal transformation (5.18). Writing the operator $Q C(R)$ of type $(1,3)$ in the form

$$
Q C(R)=R-a(\pi-2 \Phi+\Psi)-(2 a+b)(\Phi-\Psi)-(a+b+c) \Psi
$$

we shall prove

$$
Q C\left(R^{\prime}\right)=Q C(R)
$$


Taking into account (5.19) we find the relation between the curvature tensors $R^{\prime}$ and $R$ of type (1.3):

$$
\begin{aligned}
R^{\prime}-R= & -2 k \xi(u) \pi-4 k \xi(v-u) \Phi_{1}-4\left\{\xi^{2}(u)-\left(k+p^{*}\right) \xi(u)\right\} \Phi_{2} \\
& -\left\{\xi^{2}(v-u)-\left(2 k+p^{*}\right) \xi(v-u)\right\} \Psi
\end{aligned}
$$

where

$$
\begin{aligned}
& \Phi_{1}(X, Y) Z= \\
& \frac{1}{8}\{g(Y, Z)(\eta(X) \xi+\tilde{\eta}(X) J \xi)-g(X, Z)(\eta(Y) \xi+\tilde{\eta}(Y) J \xi) \\
& +g(J Y, Z)(\eta(X) J \xi-\tilde{\eta}(X) \xi)-g(J X, Z)(\eta(Y) J \xi-\tilde{\eta}(Y) \xi) \\
& -2 g(J X, Y)(\eta(Z) J \xi-\tilde{\eta}(Z) \xi)\} ; \\
& \Phi_{2}(X, Y) Z= \\
& \frac{1}{8}\{(\eta(Y) \eta(Z)+\tilde{\eta}(Y) \tilde{\eta}(Z)) X-(\eta(X) \eta(Z)+\tilde{\eta}(X) \tilde{\eta}(Z)) Y \\
& +(\eta(Y) \tilde{\eta}(Z)-\tilde{\eta}(Y) \eta(Z)) J X-(\eta(X) \tilde{\eta}(Z)-\tilde{\eta}(X) \eta(Z)) J Y \\
& -2(\eta(X) \tilde{\eta}(Y)-\tilde{\eta}(X) \eta(Y)) J Z\},
\end{aligned}
$$

$X, Y, Z \in \mathfrak{X} M$.

It follows that $\Phi_{1}+\Phi_{2}=\Phi$.

The equality (5.25) can be rewritten in the form

$$
\begin{aligned}
R^{\prime}-R= & -2 k \xi(u)(\pi-2 \Phi+\Psi) \\
& -4 k \xi(v)\left(\Phi_{1}-\frac{1}{2} \Psi\right)-4\left(\xi^{2}(u)-p^{*} \xi(u)\right)\left(\Phi_{2}-\frac{1}{2} \Psi\right) \\
& -\left(\xi^{2}(u+v)-p^{*} \xi(u+v)\right) \Psi .
\end{aligned}
$$

It is easy to check that the tensors $\pi-2 \Phi+\Psi, \Phi_{1}-\frac{1}{2} \Psi, \Phi_{2}-\frac{1}{2} \Psi$ and $\Psi$ of type $(1,3)$ change in the following way:

$$
\begin{aligned}
\pi^{\prime}-2 \Phi^{\prime}+\Psi^{\prime} & =e^{2 u}(\pi-2 \Phi+\Psi), \\
\Phi_{1}^{\prime}-\frac{1}{2} \Psi^{\prime} & =e^{2 u}\left(\Phi_{1}-\frac{1}{2} \Psi\right), \\
\Phi_{2}^{\prime}-\frac{1}{2} \Psi^{\prime} & =e^{2(u+v)}\left(\Phi_{2}-\frac{1}{2} \Psi\right), \\
\Psi^{\prime} & =e^{2(u+v)} \Psi .
\end{aligned}
$$

To prove the equality (5.24) because of (5.26) it is sufficient to prove the following equalities

$$
\begin{gathered}
-2 k \xi(u)=e^{2 u} a^{\prime}-a, \\
-4 k \xi(v)=e^{2 u}\left(2 a^{\prime}+b^{\prime}\right)-(2 a+b), \\
-4\left(\xi^{2}(u)-p^{*} \xi(u)\right)=e^{2(u+v)}\left(2 a^{\prime}+b^{\prime}\right)-(2 a+b), \\
-\left(\xi^{2}(u+v)-p^{*} \xi(u+v)\right)=e^{2(u+v)}\left(a^{\prime}+b^{\prime}+c^{\prime}\right)-(a+b+c) .
\end{gathered}
$$


Considering the equality (5.25) onto the distribution $D$ we have

$$
R^{\prime}\left(x_{0}, y_{0}\right) z_{0}=R\left(x_{0}, y_{0}\right) z_{0}-2 k \xi(u) \pi\left(x_{0}, y_{0}\right) z_{0}, \quad x_{0}, y_{0}, z_{0} \in D .
$$

Taking traces in the last equality twice we find

$$
e^{2 u}\left\{\tau^{\prime}-2 \sigma^{\prime}-2\left(\sigma^{\prime}-\varkappa^{\prime}\right)\right\}=\tau-2 \sigma-2(\sigma-\varkappa)-2 n(n-1) k \xi(u) .
$$

This equality gives (5.27) because of (2.22).

Further we apply the formula (5.17) and taking into account (4.17), (5.22) we find

$$
e^{2 u} \frac{\sigma^{\prime}-\varkappa^{\prime}}{n-1}=\frac{\sigma-\varkappa}{n-1}-k \xi(v) .
$$

The last equality becomes (5.28) because of (2.23).

From Lemma 5.4 it follows that

$$
\begin{gathered}
e^{2(u+v)} \sigma^{\prime}=\sigma-\left\{\xi^{2}(n u+v)-p^{*} \xi(n u+v)\right\}, \\
e^{2(u+v)} \varkappa^{\prime}=\varkappa-\left\{\xi^{2}(u+v)-p^{*} \xi(u+v)\right\} .
\end{gathered}
$$

Hence

$$
e^{2(u+v)} \frac{\sigma^{\prime}-\varkappa^{\prime}}{n-1}=\frac{\sigma-\varkappa}{n-1}-\left\{\xi^{2}(u)-p^{*} \xi(u)\right\}
$$

which gives (5.29).

Finally the equality (5.31) in view of (2.24) becomes (5.30).

Theorem 5.7 implies in a straightforward way that the Ricci trace $\rho(Q C(R))$ is a biconformal invariant of type $(0,2)$.

Corollary 5.8. Let $(M, g, J, D)(\operatorname{dim} M=2 n \geq 6)$ be a Kähler manifold with $B_{0^{-}}$ distribution $D$. Then the tensor of type $(0,2)$

$$
\rho-\frac{\tau-2 \sigma}{2(n-1)} g-\frac{2 n \sigma-\tau}{2(n-1)}(\eta \otimes \eta+\tilde{\eta} \otimes \tilde{\eta})
$$

is a biconformal invariant. 5.7

The following relative scalar invariant is important to the next applications of Theorem

Corollary 5.9. Let $(M, g, J, D)(\operatorname{dim} M=2 n \geq 6)$ be a Kähler manifold with $B_{0^{-}}$ distribution $D$ and $D^{\perp}=\operatorname{span}\{\xi, J \xi\}$. If $\left(g^{\prime}, \eta^{\prime}\right)$ is obtained from $(g, \eta)$ by the biconformal transformation (5.18), then

$$
a^{\prime}+k^{\prime 2}=e^{-2 u}\left(a+k^{2}\right) .
$$

Proof. From (5.27) and (5.18) we have

$$
e^{2 u} a^{\prime}=a-2 k \xi(u)=a-k^{2}\left(e^{2 v}-1\right) .
$$

On the other hand (4.17) gives

$$
e^{2 u} k^{\prime 2}=e^{2 v} k^{2}
$$

which implies the assertion. 
Thus there arise three classes of $B_{0}$-distributions, which are invariant under the biconformal group of transformations. These classes are determined by the conditions

$$
a+k^{2}>0, \quad a+k^{2}=0, \quad a+k^{2}<0,
$$

respectively.

The next question to clear up is to describe $B_{0}$-distributions in the standard flat Kähler manifold $\mathbb{C}^{n}$.

Let $\left(M, g^{\prime}, J, D\right)$ ( $\operatorname{dim} M=2 n \geq 6$ ) be a Kähler manifold with flat Levi-Chivita connection $\nabla^{\prime}$ of $g^{\prime}$ and $B_{0}$-distribution $D \quad\left(D^{\perp}=\operatorname{span}\left\{\xi^{\prime}, J \xi^{\prime}\right\}\right)$. It follows from (5.8) that

$$
\begin{gathered}
d \eta^{\prime}=0 ; \\
\nabla_{\xi^{\prime}}^{\prime} \xi^{\prime}=0 .
\end{gathered}
$$

The condition $R^{\prime}=0$ and the equalities (5.17), (5.9) imply

$$
\xi^{\prime}\left(k^{\prime}\right)+\frac{k^{\prime 2}}{2}=0
$$

Then from (5.8) and (5.35) we obtain

$$
\nabla_{x}^{\prime} \xi^{\prime}=\frac{k^{\prime}}{2} x, \quad x \in \Delta
$$

Under the conditions (5.35) and (5.36) we can describe $B_{0}$-distributions in $\mathbb{C}^{n}=\{Z=$ $\left.\left(z^{1}, \ldots, z^{n}\right)\right\}$.

Let $\left(\mathbb{C}^{n}, g^{\prime}, J, D\right)(n \geq 3)$ be the complex Euclidean space endowed with the standard flat Kähler structure $\left(g^{\prime}, J\right)$. If $D$ is a $B_{0}$-distribution in $\mathbb{C}^{n}$, then the condition (5.33) allows us to put locally $\eta^{\prime}=d t$. In this case (5.35) becomes $\frac{d k^{\prime}}{d t}=-\frac{k^{\prime 2}}{2}$ whose general solution is $k^{\prime}=\frac{2}{t+t_{0}}, t_{0}=$ const. Let us consider any integral submanifold $S^{2 n-1}$ of the integrable distribution $\Delta$. Since $\xi^{\prime}$ is a unit normal field to $S^{2 n-1}$, the condition (5.36) implies $S^{2 n-1}$ is (part of) a hypersphere with radius $r=\frac{2}{\left|k^{\prime}\right|}=\left|t+t_{0}\right|$. On the other hand according to (5.34) the integral curves of the vector field $\xi^{\prime}$ are straight lines, which pass trough the center $Z_{0}$ of $S^{2 n-1}$. Hence $S^{2 n-1}$ are concentric hyperspheres.

Choosing $Z_{0}$ as the origin $O$ of $\mathbb{C}^{n}$ we obtain the following

Canonical example of a flat Kähler manifold with $B_{0}$-distribution:

$M^{\prime}=\mathbb{C}^{n} \backslash\{O\} ;\left(g^{\prime}, J\right)$ is the standard flat Kähler structure in $\mathbb{C}^{n} ; \quad \xi^{\prime}=\frac{Z}{\|Z\|}$, where $Z$ is the position vector of the corresponding point in $M^{\prime}$.

It is easy to check that the distribution $D$ determined by $D^{\perp}=\operatorname{span}\left\{\xi^{\prime}, J \xi^{\prime}\right\}$ is a $B_{0}$-distribution and

$$
\eta^{\prime}=d r, \quad k^{\prime}=\frac{2}{r} ; \quad r^{2}=\sum_{\alpha=1}^{n} z^{\alpha} z^{\bar{\alpha}}, \quad r>0
$$


Definition 5.10. Let $(M, g, J, D)(\operatorname{dim} M=2 n \geq 6)$ be a Kähler manifold with $B_{0^{-}}$ distribution $D$ and $D^{\perp}=\operatorname{span}\{\xi, J \xi\}$. The manifold is said to be biconformally flat if there exists a flat metric $g^{\prime}$ obtained from the metric $g$ by a biconformal transformation. The metric $g$ is said to be a biconformally flat metric.

Theorem 5.11. Let $(M, g, J, D)(\operatorname{dim} M=2 n \geq 6)$ be a Kähler manifold with $B_{0^{-}}$ distribution $D$ and $D^{\perp}=\operatorname{span}\{\xi, J \xi\}$. The manifold is biconformally flat if and only if the following conditions hold good

$$
Q C(R)=0, \quad a+k^{2}>0 .
$$

Proof. Let the structure $\left(g^{\prime}, \eta^{\prime}\right)$ be obtained from the given structure $(g, \eta)$ by the biconformal transformation (5.18). If $R^{\prime}=0$, then $a^{\prime}=b^{\prime}=c^{\prime}=0$ and $Q C\left(R^{\prime}\right)=0$. According to Theorem 5.7 $Q C(R)=0$.

On the other hand Corollary 5.9 gives that

$$
a+k^{2}=e^{2 u} k^{\prime 2}>0 .
$$

For the inverse, let $Q C(R)=0$ and $a+k^{2}>0$. We shall construct a new structure $\left(g^{\prime}, \eta^{\prime}\right)$ of type (5.18), whose curvature tensor $R^{\prime}=0$.

From Corollary 5.9 and (4.17) we have

$$
e^{2 u} a^{\prime}=a-\left(e^{2 v}-1\right) k^{2} .
$$

Then the condition $a^{\prime}=0$ is equivalent to

$$
2 v=\ln \frac{a+k^{2}}{k^{2}}
$$

Lemma 5.3 and (3.21) imply that the function $v$ in (5.37) satisfies the condition $d v=$ $\xi(v) \eta$.

According to (5.8) $d \eta=0$ and we have locally $\eta=d s$ for some function $s$. Replacing $v$ from (5.37) and $\eta=d s$ into the equality

$$
2 d u=k\left(e^{2 v}-1\right) \eta
$$

we find

$$
2 u=\int \frac{a}{k} d s
$$

Let us consider the biconformal transformation (5.18) determined by the functions (5.37) and (5.38). According to Theorem 5.7 and (5.37) we have

$$
R^{\prime}=b^{\prime} \Phi^{\prime}+c^{\prime} \Psi^{\prime}
$$

Taking into account (2.23), (2.24) and $a^{\prime}=0$ the conditions $b^{\prime}=c^{\prime}=0$ are equivalent to $\sigma^{\prime}=\kappa^{\prime}=0$. According to (5.12) and (5.13) the last equalities are equivalent to the equality $k^{\prime 2}+2 k^{\prime} p^{* \prime}=0$.

From (5.28) because of (2.23) and (5.17) we have

$$
e^{2 u}\left(k^{\prime 2}+2 k^{\prime} p^{* \prime}\right)=k^{2}+2 k p^{*}-2 k \xi(v) .
$$


Calculating $2 k \xi(v)$ from (5.37) we obtain

$$
e^{2 u}\left(k^{\prime 2}+2 k^{\prime} p^{* \prime}\right)=-\frac{k}{a+k^{2}}\left\{\xi(a)+a k-k\left(k^{2}+2 k p^{*}\right)\right\}
$$

The right hand side of the last equality is identically zero because of the formulas

$$
\xi(a)=\frac{k b}{2}, \quad 2 a+b=2\left(k^{2}+2 k p^{*}\right),
$$

which follow from (3.21), (2.23) and (5.17).

Thus we proved that $a^{\prime}=b^{\prime}=c^{\prime}=0$. Hence $R^{\prime}=0$.

Theorem 5.11 allows to obtain locally all biconformally flat Kähler metrics.

Let $\left(M^{\prime}=\mathbb{C}^{n} \backslash\{O\}, g^{\prime}, J, D\right)(n \geq 3)$ be the canonical flat Kähler manifold with $B_{0}$-distribution $D$. Then

$$
r^{2}=2 g_{\alpha \bar{\beta}}^{\prime} z^{\alpha} z^{\bar{\beta}}, \quad g_{\alpha \bar{\beta}}^{\prime}=\left\{\begin{array}{cc}
\frac{1}{2} & \alpha=\beta \\
0 & \alpha \neq \beta,
\end{array} \quad \eta^{\prime}=d r, \quad k^{\prime}=\frac{2}{r} .\right.
$$

For an arbitrary function $v\left(r^{2}\right) \in C^{\infty}$ we construct the metric

$$
\begin{gathered}
g=e^{-2 u}\left\{g^{\prime}+\left(e^{-2 v}-1\right)\left(\eta^{\prime} \otimes \eta^{\prime}+\tilde{\eta}^{\prime} \otimes \tilde{\eta}^{\prime}\right)\right\} ; \\
\frac{d(-u)}{d r^{2}}=\frac{e^{-2 v}-1}{2 r^{2}},
\end{gathered}
$$

which is determined up to a constant factor (homothety).

Theorem 5.11 implies

Corollary 5.12. Let $(M, g, J, D)(\operatorname{dim} M=2 n \geq 6)$ be a Kähler manifold with $B_{0^{-}}$ distribution $D$. If the metric $g$ is biconformally flat, then it can be presented locally in the form (5.39) and vice versa.

Finally we show that the metrics (5.39) are closely related to the Kähler metrics $g$ in $\mathbb{C}^{n}$ whose potential function is $f\left(r^{2}\right) \in C^{\infty}$, i.e.

$$
g=\partial \bar{\partial} f\left(r^{2}\right)
$$

These metrics have been used as a source for Kähler metrics with special properties (cf [10, 2]).

Theorem 5.13. Let $(M, g, J, D)(\operatorname{dim} M=2 n \geq 6)$ be a Kähler manifold with $B_{0^{-}}$ distribution D. Then the metric $g$ is biconformally flat if and only if it is of type (5.40).

Proof. Let the metric $g$ be given by (5.39). Putting

$$
f\left(r^{2}\right)=\frac{1}{2} \int e^{-2 u} d r^{2}
$$

we obtain $g$ is of type (5.40).

For the inverse, let the function $f\left(r^{2}\right)$ generate the Kähler metric (5.40). Putting $e^{-2 u}=2 f^{\prime}>0, \quad e^{-2 v}=1+r^{2} \frac{f^{\prime \prime}}{f^{\prime}}>0$, we obtain the metric $g$ is of type (5.39). An easy check shows that $\xi^{\prime}(-u)=\frac{k^{\prime}}{2}\left(e^{-2 v}-1\right)$. Hence the metric $g$ is biconformally flat. QED 


\section{KäHLER STRUCTURES ON ROTATIONAL HYPERSURFACES}

The aim of this section is to show that any rotational hypersurface $\left(M^{2 n}, \bar{g}\right)$ which has no common points with the axis of revolution carries a geometrically determined complex structure $J$ and $\left(M^{2 n}, \bar{g}, J\right)$ can be considered as a locally conformal Kähler manifold. Further we show that $M^{2 n}$ carries a natural Kähler metric $g$, so that $\left(M^{2 n}, g, J\right)$ is a Kähler manifold of quasi-constant holomorphic sectional curvatures.

Let $O e$ be a fixed coordinate system in $\mathbb{R}$ and $M^{2 n}$ be a rotational hypersurface in $\mathbb{R}^{2 n+1}=\mathbb{C}^{n} \times \mathbb{R}$ with axis of revolution $l=\mathbb{R}$. We consider the class of rotational hypersurfaces having no common points with the axis of revolution. Then $M^{2 n}$ is a oneparameter family of spheres $S^{2 n-1}(s), s \in I \subset \mathbb{R}$ considered as hyperspheres in $\mathbb{C}^{n}$ with corresponding centers $q(s) e$ on $l$ and radii $t(s)>0$. If $Z$ is the radius vector of any point $p \in M^{2 n}$ with respect to the origin $O$, then the unit outer normal $n$ of the parallel $S^{2 n-1}(s)$ at the point $p$ is

$$
n=\frac{Z-q(s) e}{t(s)}
$$

Hence

$$
Z=q(s) e+t(s) n .
$$

Further we assume that $s$ is a natural parameter for the meridian

$$
\gamma(s)=q(s) e+t(s) n
$$

in the plane Oen $\left(n-\right.$ fixed), i. e. $q^{2}+t^{\prime 2}=1$.

Because of (6.2) and (6.1) the unit tangent vector field $\bar{\xi}$ to the meridian $\gamma(s)$ is

$$
\bar{\xi}=\frac{d \gamma}{d s}=q^{\prime} e+t^{\prime} n=\frac{\partial Z}{\partial s} .
$$

Since the normal to $M^{2 n}$ lies in the plane Oen, we always choose the unit vector field $N$ normal to $M^{2 n}$ by the condition that the couples $(e, n)$ and $(\bar{\xi}, N)$ have the same orientation. Then taking into account (6.3), we have

$$
N=-t^{\prime} e+q^{\prime} n \text {. }
$$

Let $\bar{g}$ be the standard metric on $\mathbb{R}^{2 n+1}=\mathbb{C}^{n} \times \mathbb{R}$ with flat Levi-Civita connection $\nabla^{\prime}$. We denote the induced metric on $M^{2 n}$ by the same letter $\bar{g}$. Then $\left(M^{2 n}, \bar{g}, \bar{\xi}\right)$ becomes the Riemannian warped product manifold [3, 5]:

$$
\gamma(s) \times_{t(s)} S_{0}^{2 n-1},
$$

where $S_{0}^{2 n-1}$ is the unit hypersphere in $\mathbb{C}^{n}$, centered at the origin $O$.

Denoting by $\bar{\nabla}$ the Levi-Civita connection on $\left(M^{2 n}, \bar{g}\right)$ we have [5]:

$$
\begin{aligned}
\bar{\nabla}_{x} \bar{\xi} & =\frac{t^{\prime}}{t} x ; \quad x \in \mathfrak{X} M^{2 n}, \bar{g}(x, \bar{\xi})=0, \\
\bar{\nabla}_{\bar{\xi}} \bar{\xi} & =0 .
\end{aligned}
$$


Let $\bar{\eta}$ be the 1 -form corresponding to the unit vector field $\bar{\xi}$ with respect to the metric $\bar{g}$, i. e. $\bar{\eta}(X)=\bar{g}(\bar{\xi}, X), X \in \mathfrak{X} M^{2 n}$. If $\bar{\pi}$ and $\bar{\Phi}$ are the tensors

$$
\begin{aligned}
\bar{\pi}(X, Y) Z= & \bar{g}(Y, Z) X-\bar{g}(X, Z) Y \\
\bar{\Phi}(X, Y) Z= & \bar{g}(Y, Z) \bar{\eta}(X) \bar{\xi}-\bar{g}(X, Z) \bar{\eta}(Y) \bar{\xi} \\
& +\bar{\eta}(Y) \bar{\eta}(Z) X-\bar{\eta}(X) \bar{\eta}(Z) Y, \quad X, Y, Z \in \mathfrak{X} M^{2 n},
\end{aligned}
$$

then the curvature tensor $\bar{R}$ of the rotational hypersurface $M^{2 n}$ has the form $[5]$ :

$$
\bar{R}=\frac{1-t^{\prime 2}}{t^{2}} \bar{\pi}-\frac{1-t^{\prime 2}+t t^{\prime \prime}}{t^{2}} \bar{\Phi}
$$

This equality implies that the rotational hypersurface $M^{2 n}$ is conformally flat.

We shall introduce a complex structure on any rotational hypersurface $M^{2 n}$ having no common points with the axis $l$.

First we consider the almost contact Riemannian structure on the parallels of the rotational hypersurface $M^{2 n}$ induced from the corresponding $\mathbb{C}^{n}$.

Let $\left(J_{0}, \bar{g}\right)$ be the standard flat Kähler structure of any $\mathbb{C}^{n}$ considered as a hyperplane in $\mathbb{R}^{2 n+1}$ perpendicular to the axis $l$. Then any parallel $S^{2 n-1}(s)$ ( $s$ - fixed) being a hypersphere in $\left(\mathbb{C}^{n}, J_{0}, \bar{g}\right)$ carries a natural almost contact Riemannian structure $(\varphi, \tilde{\bar{\xi}}, \tilde{\bar{\eta}}, \bar{g})$ determined as follows [8, 9]:

$$
\begin{aligned}
& \tilde{\bar{\xi}}=J_{0} n \\
& \tilde{\bar{\eta}}(x)=\bar{g}(x, \tilde{\bar{\xi}}), \quad x \in \mathfrak{X} S^{2 n-1}(s) ; \\
& \varphi(x)=J_{0} x+\tilde{\bar{\eta}}(x) n .
\end{aligned}
$$

The corresponding Weingarten and Gauss formulas of the imbedding $S^{2 n-1}(s) \subset \mathbb{C}^{n}$ are

$$
\begin{aligned}
\nabla_{x}^{\prime} n & =\frac{1}{t} x \\
\nabla_{x}^{\prime} y & =\tilde{\nabla}_{x} y-\frac{1}{t} \bar{g}(x, y) n ; \quad x, y \in \mathfrak{X} S^{2 n-1}(s),
\end{aligned}
$$

where $\tilde{\nabla}$ is the induced Levi-Civita connection on the sphere $S^{2 n-1}(s)$. From (6.6) and (6.7) it follows directly that

$$
\begin{aligned}
& \tilde{\nabla}_{x} \tilde{\bar{\xi}}=\frac{1}{t} \varphi x, \quad x \in \mathfrak{X} S^{2 n-1}(s) ; \\
0=\left(\nabla_{x}^{\prime} J_{0}\right) y= & \left(\tilde{\nabla}_{x} \varphi\right) y-\frac{1}{t} \tilde{\bar{\eta}}(y) x+\frac{1}{t} \bar{g}(x, y) \tilde{\bar{\xi}} \\
& -\left(\frac{1}{t} \bar{g}(x, \varphi y)+\left(\tilde{\nabla}_{x} \tilde{\bar{\eta}}\right) y\right) n, \quad x, y \in \mathfrak{X} S^{2 n-1}(s) .
\end{aligned}
$$


Hence

$$
\left(\tilde{\nabla}_{x} \varphi\right) y=\frac{1}{t}(\tilde{\bar{\eta}}(y) x-\bar{g}(x, y) \tilde{\bar{\xi}}), \quad x, y \in \mathfrak{X} S^{2 n-1}(s)
$$

If the structure $(\varphi, \tilde{\bar{\xi}}, \tilde{\bar{\eta}}, \bar{g})$ of an almost contact Riemannian manifold satisfies the conditions

$$
\begin{aligned}
& \tilde{\nabla}_{x} \tilde{\bar{\xi}}=\alpha \varphi x, \quad \alpha=\text { const } \\
& \left(\tilde{\nabla}_{x} \varphi\right) y=\alpha(\tilde{\bar{\eta}}(y) x-\bar{g}(x, y) \tilde{\bar{\xi}})
\end{aligned}
$$

then the manifold is called an $\alpha$-Sasakian manifold $[\underline{6}$. In the case $\alpha=1$ these manifolds are the usual Sasakian manifolds.

Taking into account the equalities (6.8) and (6.9) we conclude that the structure (6.6) on any hypersphere $S^{2 n-1}(s)\left(s\right.$ - fixed) in $\mathbb{C}^{n}$ is $\frac{1}{t}$-Sasakian $\left(\frac{1}{t}=\alpha=\right.$ const $)$.

Now we can introduce a complex structure $J$ on the rotational hypersurface $M^{2 n}$ subordinated to the orientation $\bar{\xi}$ of the meridians.

Let $T_{p} M^{2 n}$ be the tangent space to $M^{2 n}$ at any point $p$. Then the vector fields $\bar{\xi}$ and $\tilde{\bar{\xi}}$ defined by (6.6) determine a distribution $D$ so that $D^{\perp}=\operatorname{span}\{\bar{\xi}, \tilde{\bar{\xi}}\}$. We define the almost complex structure $J$ associated with $\bar{\xi}$ as follows:

$$
J_{\mid D}=J_{0}, \quad J \bar{\xi}=\tilde{\bar{\xi}}, \quad J \tilde{\bar{\xi}}=-\bar{\xi}
$$

It is clear that $J$ is an almost complex structure and $\left(M^{2 n}, \bar{g}, J\right)$ becomes an almost Hermitian manifold.

The almost complex structure $J$ defined by (6.10) and the structure $\varphi$ on $S^{2 n-1}(s)$ given by (6.6) are related as follows:

$$
\varphi(x)=J x+\tilde{\bar{\eta}}(x) \bar{\xi}, \quad x \in \mathfrak{X} S^{2 n-1}(s) .
$$

Below we give the Weingarten and Gauss formulas for the embedding $S^{2 n-1}(s) \subset M^{2 n}$ with normal vector field $\bar{\xi}$ :

$$
\begin{aligned}
& \bar{\nabla}_{x} \bar{\xi}=\frac{t^{\prime}}{t} x, \\
& \bar{\nabla}_{x} y=\tilde{\nabla}_{x} y-\frac{t^{\prime}}{t} \bar{g}(x, y) \bar{\xi}, \quad x, y \in \mathfrak{X} S^{2 n-1}(s) .
\end{aligned}
$$

The Gauss formula and (6.8) imply immediately that

$$
\bar{\nabla}_{x} \tilde{\bar{\xi}}=\frac{1}{t} \varphi x-\frac{t^{\prime}}{t} \tilde{\bar{\eta}}(x) \bar{\xi}, \quad x \in \mathfrak{X} S^{2 n-1}(s) .
$$

Further we prove

Proposition 6.1. Let $(M, \bar{g})$ be a rotational hypersurface in $\mathbb{C}^{n} \times R$ with axis of revolution $l=\mathbb{R}$, which has no common points with $l$ and the meridians of $M^{2 n}$ are oriented with 
the unit vector field $\bar{\xi}$. If $J$ is the almost complex structure (6.10) associated with $\bar{\xi}$, then the covariant derivative of $J$ satisfies the identity

$$
\left(\bar{\nabla}_{X} J\right) Y=\frac{t^{\prime}-1}{t}(\bar{g}(X, Y) \tilde{\bar{\xi}}-\tilde{\bar{\eta}}(Y) X-\bar{\eta}(Y) J X+\bar{g}(J X, Y) \bar{\xi})
$$

for all vector fields $X, Y \in \mathfrak{X} M^{2 n}$.

Proof: To prove (6.14) it is sufficient to show that the following equalities hold good:

(i) $\left(\bar{\nabla}_{\bar{\xi}} J\right) x=0, \quad \bar{g}(\bar{\xi}, x)=0 ;$

(ii) $\left(\bar{\nabla}_{\bar{\xi}} J\right) \bar{\xi}=0$;

$$
\begin{aligned}
& (i i i)\left(\bar{\nabla}_{x} J\right) y=\frac{t^{\prime}-1}{t}(\bar{g}(x, y) \tilde{\bar{\xi}}-\tilde{\bar{\eta}}(y) x+\bar{g}(\varphi x, y) \bar{\xi}), \quad \bar{g}(\bar{\xi}, x)=\bar{g}(\bar{\xi}, y)=0 ; \\
& (i v)\left(\bar{\nabla}_{x} J\right) \bar{\xi}=\frac{1-t^{\prime}}{t}(J x+\tilde{\bar{\eta}}(x) \bar{\xi}), \quad \bar{g}(\bar{\xi}, x)=0 .
\end{aligned}
$$

The equality (i) follows because of the fact that the hyperplanes $\mathbb{C}^{n}(s)$ are parallel along any meridian $\gamma(s)$.

Taking into account (i) and (6.4)it follows that (ii) holds good.

The equality (iii) follows from (6.12), (6.13), (6.11) and (6.9).

Finally (iv) follows from (6.12), (6.13) and (6.11).

QED

The identity (6.14) implies that the almost complex structure $J$ is integrable, i. e. $\left(M^{2 n}, \bar{g}, J\right)$ is a Hermitian manifold. Moreover, this manifold is in the class $W_{4}$ according to the classification in [4. A simple computation shows that the Lee form of the manifold is $\frac{1-t^{\prime}}{t} \bar{\eta}$. From (6.4) it follows that the 1 -form $\bar{\eta}$ is closed and therefore the Lee form of $\left(M^{2 n}, \bar{g}, J\right)$ is also closed. Then the manifold under consideration is locally conformal Kähler in all dimensions $2 n \geq 4$. This implies $\left(M^{2 n}, \bar{g}, J\right)$ carries a conformal Kähler metric which is flat by virtue of the fact that $\bar{g}$ is conformally flat.

Our aim is to define another nontrivial Kähler metric on $\left(M^{2 n}, \bar{g}, J\right)$, which is naturally determined by its geometric structures.

We constrain the class of the rotational hypersurfaces which have no common points with the axis of revolution, assuming the inequality $t^{\prime}(s) \neq 0, s \in I$. This condition means that the meridian $\gamma(s)$ of $M^{2 n}$ has no points in which the tangents are parallel to the axis $l$. Under the condition $t^{\prime} \neq 0$ we can choose in a unique way the orientation $\bar{\xi}$ of the meridians so that $t^{\prime}(s)>0$.

In what follows we consider the class of rotational hypersurfaces $\left(M^{2 n}, \bar{g}, J\right)$ satisfying the following inequalities:

$$
t(s)>0, \quad t^{\prime}(s)>0 ; \quad s \in I .
$$

Under the conditions (6.15) we construct the metric

$$
g=\bar{g}+\left(t^{\prime}-1\right)(\bar{\eta} \otimes \bar{\eta}+\tilde{\bar{\eta}} \otimes \tilde{\bar{\eta}}) .
$$

Taking into account (6.8) and (6.11) it is easy to check that the Kähler form of the metric $g$ is closed, i. e. $g$ is a Kähler metric. We call $g$ a complex dilatational Kähler 
metric on the rotational hypersurface $M^{2 n}$. This metric is relevant to the subject of our considerations in the present paper because of the following

Theorem 6.2. Let $\left(M^{2 n}, \bar{g}, J, \bar{\xi}\right)(2 n \geq 4)$ be a rotational hypersurface satisfying the conditions (6.15). Then the complex dilatational Kähler metric g given by (6.16) is of quasiconstant holomorphic sectional curvatures.

Proof: Let $\nabla$ be the Levi-Civita connection of the metric (6.16). Using (6.4) and (6.13) we find in a standard way that

$$
\begin{aligned}
\nabla_{X} Y= & \bar{\nabla}_{X} Y+\frac{1-t^{\prime}}{t}\{\bar{\eta}(J X) J Y+\bar{\eta}(J Y) J X-\bar{g}(X, Y) \bar{\xi}\} \\
& +\left(\frac{1-t^{\prime}}{t}+\bar{\xi}\left(\ln \sqrt{t^{\prime}}\right)\right)\{\bar{\eta}(X) \bar{\eta}(Y)-\bar{\eta}(J X) \bar{\eta}(J Y)\} \bar{\xi} \\
& -\left(\frac{1-t^{\prime}}{t}+\bar{\xi}\left(\ln \sqrt{t^{\prime}}\right)\right)\{\bar{\eta}(X) \bar{\eta}(J Y)+\bar{\eta}(J X) \bar{\eta}(Y)\} J \bar{\xi}
\end{aligned}
$$

for all $X, Y \in \mathfrak{X} M^{2 n}$.

Taking into account (6.17), (6.5) and (6.16) we compute the curvature tensor $R$ of the connection $\nabla$ :

$$
R=a \pi+b \Phi+c \Psi
$$

where

$$
a=\frac{4\left(1-t^{\prime}\right)}{t^{2}}, \quad b=8\left(\frac{t^{\prime}-1}{t^{2}}-\frac{t^{\prime \prime}}{2 t t^{\prime}}\right), \quad c=\frac{4\left(1-t^{\prime}\right)}{t^{2}}+\frac{5 t^{\prime \prime}}{2 t t^{\prime}}+\frac{t^{\prime \prime 2}-t^{\prime} t^{\prime \prime \prime}}{2 t^{\prime 3}}
$$

Applying Proposition 2.3 we obtain the assertion.

QED

Since $t^{\prime 2}=1-q^{2}$, then $t^{\prime} \in(0,1]$. Hence $a \geq 0$ in (6.18).

From the equality $q^{2}=1-t^{\prime 2}$ it also follows that the function $t=t(s)$ determines the rotational hypersurface $M^{2 n}$ up to a translation along the axis $l$ and a symmetry with respect to the hyperplane $\mathbb{C}^{n}$ through the origin $O$.

Let $p \in M^{2 n}$ and $\gamma(p), S^{2 n-1}(p)$ be the corresponding meridian and parallel through the point $p$. From (6.14), (6.5) and (6.18) it follows that the following conditions are equivalent:

(1) the tangent $\bar{\xi}$ to $\gamma$ at $p \in M^{2 n}$ is perpendicular to the axis $l$;

(2) $g=\bar{g}$ on the parallel $S^{2 n-1}(p)$ through the point $p$;

(3) $\bar{\nabla} J=0$ on $S^{2 n-1}(p)$;

(4) $\bar{R}=0$ on $S^{2 n-1}(p)$;

(5) $R=0$ on $S^{2 n-1}(p)$.

As a consequence of Theorem 6.2 we can find the rotational hypersurfaces $M^{2 n}$ whose complex dilatational Kähler metric is of constant holomorphic sectional curvatures. 
Let $b=0$ in (6.18). Then Corollary 3.6 implies that $c=0$ and the metric $g$ is of constant holomorphic sectional curvature $a=$ const $>0$.

Solving the equation

$$
b=8\left(\frac{t^{\prime}-1}{t^{2}}-\frac{t^{\prime \prime}}{2 t t^{\prime}}\right)=0
$$

we obtain the meridian in the form $q=q(t)$.

Considering the meridian in the usual coordinate system $O x y$ with axis of revolution $l=O y$ we have:

Proposition 6.3. Any rotational hypersurface $M^{2 n}$ which carries a complex dilatational Kähler metric of constant holomorphic sectional curvature $a=$ const $>0$ is generated by a meridian of the type

$$
\begin{gathered}
\gamma: y= \pm \frac{1}{\sqrt{a}}\left(\sqrt{8-a x^{2}}+\ln \frac{\sqrt{8-a x^{2}}-2}{\sqrt{8-a x^{2}}+2}\right)+y_{0}, \quad 0<x<\frac{2}{\sqrt{a}} . \\
\text { REFERENCES }
\end{gathered}
$$

[1] Boju, V.; Popesku, M. Espaces à courbure quasi-constante, J. Diff. Geom., 13 (1978), 373-383.

[2] Bryant, R. Bochner-Kähler metrics, J. Amer. Math. Soc., 14 (2001), 623-715.

[3] Bishop, R.; O'Neil, B. Manifolds of negative curvature, Trans. Amer. Math. Soc., 145 (1969), 1-49.

[4] Gray, A.; Hervella, L. The sixteen classes of almost Hermitian manifolds and their linear invariants, Ann. di Mat. Pura ed Appl., 123 (1980), 35-58.

[5] Ganchev, G.; Mihova, V. Riemannian manifolds of quasi-constant sectional curvature, J. reine und angew. Math., 522 (2000), 119-141.

[6] Janssens, D.; Vanhecke, L. Almost contact structures and curvature tensors, Kodai Math. J., 4 (1981), 1-27.

[7] Kobayasi, S.; Nomizu, K. Foundations of Differential Geometry, II, Interscience Publishers, New Yourk, 1969.

[8] Tashiro, Y. On contact structures on hypersurfaces in almost complex manifolds I. Tohoku Math. J., bf 15 (1963), 62-79.

[9] Tashiro, Y. On contact structures on hypersurfaces in almost complex manifolds II. Tohoku Math. J., bf 15 (1963), 167-175.

[10] Tachibana, S.; Liu, R.C. Notes on Kählerian metrics with vanishing Bochner curvature tensor, Kodai Math. Sem. Rep., 22 (1970), 313-321.

[11] Tricerri, F.; Vanhecke, L. Curvature tensors on almost Hermitian manifolds, Trans. Amer. Math. Soc., 267 (1981), 365-398.

Bulgarian Academy of Sciences, Institute of Mathematics and Informatics, Acad. G. Bonchev Str. BL. 8, 1113 Sofia, Bulgaria

E-mail address: ganchev@math.bas.bg

Faculty of Mathematics and Informatics, University of Sofia, J. Bouchier Str. 5, (1164) Sofia, Bulgaria

E-mail address: mihova@fmi.uni-sofia.bg 